\title{
III. Verhalten gegenüber der Zivilbevölkerung, innere Konflikte, Disziplin
}

\section{Einleitung}

In der sowjetischen Historiographie heißt es, sowjetische Partisanen seien unter der Bevölkerung sehr beliebt gewesen und hätten stets auf ihre Unterstützung zählen können. Die Partisanen ihrerseits hätten die Bevölkerung vor Repressionen der deutschen Besatzer geschützt und sie korrekt behandelt. In zeitgenössischen offiziellen Berichten an die Zentrale schilderte die Führung des Verbandes Baranoviči das Verhältnis der einheimischen Bevölkerung zu den sowjetischen Partisanen komplexer. Danach habe sich die Bevölkerung, allerdings mit Ausnahme der polnischen, gegenüber den sowjetischen Partisanen insgesamt positiv verhalten. General „Platon" berichtete im Oktober 1943 folgendermaßen an Ponomarenko:

Es sei hervorgehoben, daß die Belorussen den Partisanen positiv gegenüberstehen und sich den Abteilungen anschließen, während sich viele Polen negativ zu den Partisanen verhalten und ihnen sogar feindlich gesinnt sind. Es ist eine Reihe von Fällen bekannt, in denen Polen in den Dörfern kleine Partisanengruppen bei der Ausführung von Aufträgen ermordet haben. Sie benachrichtigen die Deutschen über die Bewegungen der Partisanen. Die katholischen Belorussen schwanken und verhalten sich reserviert, einerseits sympathisieren sic mit den Partisanen, aber ein Teil von ihnen arbeitet mit den Polen zusammen. Unter solchen Leuten leisten wir die entsprechende Arbeit. ${ }^{1}$

Aber auch diese Schilderung gab die historische Realität nicht ganz korrekt wieder, wenigstens was die Verhältnisse in der Oblast' Baranoviči anbetrifft.

Im Sommer 1942 berichtete die "Korweta“-Abteilung der Heimatarmee, daß die sowjetische Partisanenbewegung in den ehemaligen östlichen Teilen Polens sehr stark gewachsen sei, und daß sich als Nebenerscheinung das Banditentum stark entwickelt habe: „Die Tatsache, daß in diesen Gebieten sowjetische Partisanen grassieren, nutzen ad hoc gebildete Banden, die zu der schlimmsten Plage in dieser Gegend wurden. Gut bewaffnete Draufgänger überfallen meistens Gemeindekassen, Güter und seltener wohlhabende Bauern." 2

Mit der Zeit verschlechterte sich die Lage. In einem Bericht der Heimatarmee für den Bezirk Novogródek heißt es: „Im vergangenen Jahr [1943] verbreiteten im Bezirk zahlreiche sowjetische Partisanen und Räuberbanden Angst und Schrecken.

1 Ausführlicher Bericht über den Zustand des Untergrundes und der Partisanenbewegung in der Oblast' Baranoviči zum 1. 10. 1943: Kapitel I., Dokument Nr. 8, S. 58.

2 „Korweta“: Bericht in der Angelegenheit „ $\mathrm{K}$ “ für die Zeit vom 20. 6.-20. 7. 1942: AAN, MF 423, Bl. 57. Tatsächlich waren jedoch die Überfälle auf Güter und Gemeinden auch eine gezielte Aktionsform der sowjetischen Partisanen (vgl. Kapitel II.). 
Eher selten setzten sie den Deutschen zu, die sich unwillig in die von den Banden beherrschten Gebiete begaben, um jene zu liquidieren. "3 In einem anderen Bericht der Heimatarmee vom September 1943 steht: „Die Landbevölkerung geriet buchstäblich zwischen Hammer und Amboß, einerseits leidet sie unter dem Terror der Partisanen, anderseits unter den Repressionen der Deutschen und der Weißen Milizen."4

Diese Berichte mögen übertrieben erscheinen und eher die antisowjetischen Vorurteile der Führung der Heimatarmee bzw. der polnischen Bevölkerung wiedergeben als die damalige Wirklichkeit. Im Kern bestätigen jedoch zeitgenössische sowjetische Berichte diese Vorwürfe. Der Partisan Rudenko von der Brigade „Nikitin“ beschrieb das Verhalten zahlreicher Partisanengruppen und -abteilungen im Jahre 1942 auf dem Gebiet des westlichen Weißrußland wie folgt:

Da sie [Partisanen] nicht kämpfen, arten sie unvermeidlich zu einer zusätzlichen Last für die Bauern aus und bringen die Bauernschaft gegen die Partisanen insgesamt auf. Folglich schadet das der Partei und Regierung; konkret geschieht das folgendermaßen: Wenn die Deutschen nicht da sind, gehen die Partisanen ungehindert im Dorf hin und her, leisten keine Massenarbeit unter den Bauern, nehmen ihnen Kühe, Schafe, Brot und andere Lebensmittel weg. Sobald aber eine Strafabteilung auftaucht, fliehen die Partisanen, ohne Widerstand zu leisten, während die Bauern dann geprügelt und ihre Häuser in Brand gesteckt werden, weil sie die Partisanen unterhalten und verpflegt haben. Die Bauern sagen ohne Umschweife: „Zum Kuckuck mit solchen Partisanen, die mir das Letzte wegnehmen und mich nicht gegen die Deutschen verteidigen können. Ohne euch hätte der Deutsche mich nicht geprügelt und nicht mein Haus in Brand gesteckt." 5

In einem anderen sowjetischen Bericht aus dieser Zeit (Winter 1942/43) wird die fehlende einheitliche Führung der Partisanenbewegung in der Oblast' Baranoviči bemängelt, die dazu führe, daß sich eine Reihe von kleineren Partisanengruppen und teilweise großen Abteilungen passiv verhalte. Anstatt mit dem Feind zu kämpfen, raubten sie die Bevölkerung aus, so daß diese gegen alle Partisanen aufgebracht sei. Die Deutschen nutzten die Gelegenheit und stellen in den Dörfern Selbstverteidigungsgruppen (Samochova) auf, die ausschließlich gegen die Partisanen kämpften. ${ }^{6}$

Exemplarisch ist das Schicksal der Ortschaft Naliboki. In der Nacht zum 8. Mai 1943 überfielen sowjetische Partisanen den dortigen Stützpunkt der "Samochova“, töteten u.a. 127 Zivilisten, brannten zahlreiche Gebäude nieder und erbeuteten bis zu 100 Kühe und 70 Pferde. ${ }^{7}$ Zwei Monate später, während des Unternehmens „Hermann“ (13. Juli - 8. August 1943), brannte die deutsche Strafexpedition die ganze Ortschaft nieder. Dabei ermordeten die Deutschen etwa 10 Einwohner, darunter zwei katholische Geistliche, den Rest der Bevölkerung (etwa 3000) ver-

3 Militärische Lage im Bezirk „Now“ [Nowogródek], 19. 6. 1944: AAN, 203/VII-56, Bl. 4.

4 Lagebericht der AK für die Wojewodschaft Nowogródek, Baranowicze, 5.9. 1943: AAN, Mikrofilm M- B582/1-2, Fondas R-601, apyrasas 1, vien.: 27, Bl. 3-5, hier Bl. 4.

5 Aufzeichnungen des Partisanen Je. D. Rudko aus der Brigade "Nikitin“ (ohne Datum, wahrscheinlich Winter 1942/43): Siehe Kapitel I., Dokument Nr. 4, S. 52.

6 Bericht über die Kampfaktivitäten der 125. Stalin Partisanenabteilung von der Entstehung bis Ende 1942: NARB, f. 4p, op. 33a, d. 169, 11. 31-37.

7 Siehe Kapitel II., Dokumente Nr. 2 und 3. 
schleppten sie zur Zwangsarbeit und erbeuteten das übriggebliebene lebende Inventar. ${ }^{8}$

Wie oben gezeigt, waren sich einige Partisanenführer dessen bewußt, daß sich die Bevölkerung in einer sehr kritischen Lage befand. Manche versuchten bereits im Jahre 1942 dagegen vorzugehen und die Bevölkerung vor Übergriffen eigener Partisanen und krimineller Banden zu schützen. Beispielsweise stellten Gromov und seine Partisanen, die im Sommer 1942 in der Region um Novogrudok operierten, bei Requirierungen von Lebensmitteln Bescheinigungen aus. Sonst verhielten sie sich der Zivilbevölkerung gegenüber freundlich und gingen gegen kriminelle Banden vor. ${ }^{9}$ Gromov wurde jedoch bald von einem sowjetischen Partisanen getötet. ${ }^{10}$ Das Verhalten von Gromov und seiner Partisanen, die offenkundig tatsächlich vor allem mit den deutschen Besatzern kämpften ${ }^{11}$, scheint jedoch zu dieser Zeit eher eine Ausnahme als die Regel gewesen zu sein.

Im Jahre 1943 sah sich die Führung des Verbandes Baranoviči veranlaßt, den Kampf gegen das Marodieren, exzessiven Alkoholmißbrauch, Gewalttätigkeiten und Disziplinlosigkeit in den eigenen Reihen auszurufen. ${ }^{12}$ Die Methoden, mit denen man dagegen vorging, waren verschieden. Sie reichten von Appellen, Verboten, Strafandrohungen, über exemplarische Bestrafung bis hin zur Auflösung besonders demoralisierter Abteilungen.

Im Frühjahr 1943 beschloß die Führung des Verbandes Ivenec, die Gruppe Boženko aus der Brigade „Žukov" aufzulösen und Boženko vor ein Partisanengericht zu stellen, denn: „Die Gruppe Boženko [...] befaßte sich mit Saufgelagen, Plünderung und sie terrorisierte die friedliche Bevölkerung. [...] Sie führte keine bewaffneten Aktionen durch. "13 Auch eine ganze Brigade war davon betroffen, als im Mai 1943 die Führung des Verbandes Ivenec die Brigade „Frunze“ auflösen und sie unter einer neuen Führung umorganisieren ließ. Die Partisanenbrigade „Frunze“ zerfiel im Februar 1943 nach einer deutschen Strafexpedition und operierte bis Mai in einzelnen Gruppen. „Manche Gruppen begannen, sich unangemessen zu verhalten - sie marodieren, saufen und mißhandeln die Bevölkerung. “14

Um willkürliche Requirierungen einzudämmen und unter Kontrolle zu bringen, ließ die Verbandsführung Baranoviči, Operationsgebiete der einzelnen Brigaden in Zonen aufteilen, in denen sich nur bestimmte Abteilungen versorgen durften. Die Abgaben von Getreide, Fleisch und anderen Lebensmitteln an Partisanen waren für jedes Dorf bzw. jedes Anwesen festzulegen, wobei örtliche Bedingungen zu be-

8 Siehe Kapitel II., Dokument Nr. 5.

9 „Korweta“ Bericht über die Aktivitäten „K“ für die Zeit vom 20.6. bis zum 20. 7. 1942: AAN, MF 423, Bl. 89.

10 Entstehungsgeschichte der Abteilungen der Brigade „Pervomajskaja“: NARB, f. 3500, op. 4, d. 250, 11. 89-121; abgedruckt in Kapitel I., Dokument Nr. 16, S. 91.

11 Siehe dazu die „Korweta“ Berichte für das Jahr 1942: AAN, MF 423, Bl. 81, 89, $356 f$.

12 Siehe Kapitel II., Dokument Nr. 5.

13 Bericht des Bevollmächtigten des ZK der $\mathrm{KP}(\mathrm{b}) \mathrm{B}$ in der Rajon Ivenec Budaj über militärische Aktivitäten der Brigade „Žukov" und der Kosaken-Abteilung vom 8. 7. 1943: NARB, f. 1329, op. 1, d. 32, 1l. 13f; zu Boženko siehe auch: Kapitel IV., Dokument Nr. 2.

14 Befehl Nr. 11 des Bevollmächtigten des ZK der KP(b)B und des BŠDP an vereinigtes Rajonzentrum Ivenec, Dubov, vom 9. 5. 1943: NARB, f. 1329, op. 1, d. 30, 1. 14; siehe auch Kapitel I., Dokument Nr. 6. 
rücksichtigen waren. Willkürliches Requirieren wurde verboten. ${ }^{15}$ Die Führung des Vereinigten Rajonzentrums Ivenec ließ in ihrem Operationsgebiet zwölf Partisanenkommandanturen einrichten, um das Marodieren, Vergewaltigungen und andere Übergriffe der Partisanen einzudämmen. ${ }^{16}$

Man setzte auch auf parteipolitische Massenarbeit in den Abteilungen. Der Kommissar der Brigade „Žukov“" meldete im Dezember 1943: „Intensivierung der politischen Arbeit in der Brigade ermöglichte in kurzer Zeit, Saufgelage, Marodieren, Gewalttaten und nicht sowjetisches Verhalten gegenüber der friedlichen Bevölkerung zu unterbinden. Infolgedessen erhöhte sich die Autorität der Partisanen in den Ortschaften." 17 Zahlreiche andere Dokumente, die hier zum Teil veröffentlicht sind, deuten jedoch daraufhin, daß dies eine typische, d.h. allzu optimistische Erfolgsmeldung ist. Denn der Kampf gegen diese Erscheinungen erfolgte oft nur auf dem Papier und in der Praxis änderte sich offenkundig nicht viel.

In einem Befehl vom 14. Januar 1944 rügte die Führung des Vereinigten Rajonzentrums Ščučin ihre Abteilungskommandeure: „Einzelne Kommandeure und Kommissare haben gegen die außer Rand und Band geratenen Marodeure und Säufer keine entschlossenen Maßnahmen getroffen, ganz im Gegenteil: Sie waren bemüht, den wirklichen Sachverhalt zu verheimlichen, beschränkten sich auf Gespräche und bewogen dadurch die zersetzenden Elemente dazu, ihre schändlichen Handlungen gegenüber der örtlichen Bevölkerung fortzusetzen." 18

Am 3. Februar 1944 tadelte der Stellvertreter von General „Platon“, Carjuk, den Kommandeur der Abteilung „Erster Komsomol“, A. Sajatevič: „Trotz der wiederholten und kategorischen Befehle, einen entschiedenen Kampf gegen Gewalt, Saufgelage und widerrechtliche Behandlung der friedlichen Bevölkerung zu führen, begehen Partisanen ihrer Abteilungen diese Vergehen. "19 Einen Monat später, am 6. März 1944, kritisierte der stellvertretende Bevollmächtigte des ZK der KP(b)B in der Oblast' Baranoviči, Donskoj, scharf die weiterhin auftretenden schwerwiegenden Verstöße gegen die Partisanendisziplin, und forderte die Chefs der NKVDSonderabteilungen bei den Brigaden des Verbandes auf, gegen Marodieren, Trunkenheit, Gewalt gegen die Zivilbevölkerung und andere Verstöße gegen die Disziplin entschieden vorzugehen. ${ }^{20}$

Ein großes Problem stellte die Trunkenheit der Partisanen dar. Partisanen betranken sich oft und begingen in diesem Zustand Gewalttaten, in der Regel an Zivilisten aber auch an eigenen Kameraden. So tötete beispielsweise der Kommandeur der Abteilung „Ponomarenko“ (Brigade „Džeržinski“) Nikolaj Kajdanov am 5. Dezember 1943 im alkoholisierten Zustand einen Partisanen der Abteilung „Ždanov“ derselben Brigade. In einem Bericht dazu heißt es: „Dieser Fall ist nicht sein erster.

15 Siehe Kapitel III., Dokument Nr. 3.

16 Siehe Kapitel III., Dokument Nr. 4 vom 21. 7. 1943.

17 Tätigkeitsbericht des Politkommissars der Brigade Žukov, Gajsarov, für die Zeit vom 1.10. bis zum 15. 12. 1943, 21. 12. 1943: NARB, f. 1329, op. 1, d. 69, 11. 16-21.

18 Siehe Kapitel III., Dokument Nr. 7, S. 170.

19 Schreiben des stellvertretenden Bevollmächtigen des BŠPD und des ZK der KP(b)B, Carjuk, vom 3. Febraur 1944: NARB, f. 1329, op. 1, d. 72, 1. 43.

20 Schreiben des stellvertretenden Bevollmächtigten des ZK der KP(b)B in der Oblast' Baranoviči, Donskoj, an alle Chefs der NKVD-Sonderabteilungen bei den Brigaden des Verbandes Baranoviči vom 6. 3. 1944: NARB, f. 1329, op. 1, d. 51, ll. $166 \mathrm{f}$. 
Im Monat November d. J. trank er Alkohol mit einer Gruppe von Partisanen im zivilen Waldlager in der Nähe von Kletišče. Als er sich völlig betrunken hatte, entkleidete er Personen aus dem Lager, nahm ihnen die Kleider weg und schoß auf sie aus einer Maschinenpistole." 21

Alkohol „besorgten sich“ bzw. erpreßten die Partisanen meistens bei den Bauern. Oft „requirierten“ die Partisanen Pferde, Schafe, Vieh, Kleidungsstücke und verschiedene Haushaltsgegenstände, die sie später in anderen Ortschaften veräußerten, um vom Erlös Alkohol zu kaufen, oder auch gleich gegen Alkohol einzutauschten.

Die hier publizierten Dokumente zeigen, daß die Disziplin in den einzelnen Partisanenabteilungen trotz der Bemühungen der Führung bis zum Schluß relativ schlecht war. Zwischen den einzelnen Partisanen, Gruppen und selbst Abteilungen kam es oft zu Streitigkeiten, die nicht selten mit Waffen ausgetragen wurden, und die nicht nur Opfer unter den Partisanen, sondern auch unter unbeteiligten Zivilisten verursachten. Auch das Verhalten der sowjetischen Partisanen gegenüber der Zivilbevölkerung, insbesondere der polnischen ${ }^{22}$, blieb bis zum Einmarsch der Roten Armee im Juli 1944 unverändert schlecht. Die Ernährungslage der Bauern wurde dagegen immer kritischer.

21 Aktennotiz der Führung des Vereinigten Rajonzentrums Lida Nr. 008 vom 25. 12. 1943: NARB, f. 1329, op. 1, d. 51, 11. 100.

22 Siehe Kapitel V. 


\section{Dokumente}

\section{Dokument Nr. 1}

Befehl Nr. 004 vom 22. Mai 1943 des Bevollmächtigten des Belorussischen Stabs der Partisanenbewegung und des ZK der KP(b)B für das vereinigte Rajonzentrum Lida über das Saufen und ziellose Schießen im Dorf Potašnja

Befehl

des Bevollmächtigten des Belorussischen Stabs der Partisanenbewegung und des $Z K \operatorname{der} K P(b)$

für das vereinigte Rajonzentrum Lida

Nr. 004 vom 22. Mai 1943

I.

In der Zeit meines Aufenthaltes in der Aleksandr-Nevskij-Abteilung wurde festgestellt, daß einzelne Kommandeure wiederholt in der Abteilung Saufgelage mit ihren Unterstellten veranstalten, wobei in der Regel aus persönlichen Waffen geschossen wird. Das führt zum Nachlassen der Wachsamkeit, zur Zersetzung der Disziplin, zur Minderung der Kampffähigkeit der Abteilung sowie zur Unterminierung ihrer Autorität in der Bevölkerung. Ein Beispiel: Der Stabschef der AleksandrNevskij-Abteilung, Gen. V. P. Bogatyrev, veranstaltete am 27. April dieses Jahres im Dorf Potašnja, in einer Wohnung, in der sich die Verhaftete Sovčik (später erschossen) befand, in Anwesenheit von Unterleutnant Somin und von Leutnant Smirin aus der Kutuzov-Abteilung ein Saufgelage.

Die Zurechtweisung meinerseits und seitens des Abteilungskommandeurs Bojkov und die Forderung, mit dem Saufen aufzuhören und nicht zur Verhafteten hineinzugehen, wurde von Bogatyrev mißachtet. Da er betrunken war, überhörte er unter Mißbrauch seiner Dienstbefugnisse als Stabschef die Warnung des Wachpostens, daß es verboten sei, zur Verhafteten hineinzugehen und dort ein Saufgelage zu veranstalten.

Am 3. Mai begann Gen. Bogatyrev, der alkoholisiert war, hinter dem Dorf Potašnja mit seinem Unterstellten Fillipovič eine lang andauernde Schießerei aus einem Gewehr und einer Maschinenpistole.

Obwohl ich ihn persönlich zweimal verwarnte und obwohl er versprach, sich zu bessern, betrank sich Gen. Bogatyrev am 17. Mai im Dorf Potašnja selbst, machte auch seinen Unterstellten Visoulov betrunken und eröffnete im selben Haus wieder das Feuer, dann schoß er weiter im Dorf und auf der Wiese und erschien in Standortbereich des Lagers erst um 20 Uhr des 18. Mai.

II.

Wegen des wiederholten Alkoholmißbrauchs und der Verleitung seiner Unterstellten zum Saufen sowie wegen der ziellosen Schießerei unter Alkoholeinwirkung 
erteile ich Gen. Bogatyrev, Stabschef der Aleksandr-Nevskij-Abteilung, eine Rüge mit Verwarnung. Sollten sich ähnliche Vorkommnisse in der Zukunft wiederholen, so wird das als vorsätzlicher Mißbrauch betrachtet und werden strengere Maßnahmen getroffen werden.

III.

Ich verpflichte den Kommandeur der Abteilung Gen. A. G. Bobkov, genau festzustellen, unter welchen Umständen es zum Schießen in der Wohnung kam und welchen Schaden Bogatyrev durch die Schießerei den Bewohnern dieses Hauses zugefügt hat.

IV.

Das Abteilungskommando hat alle Maßnahmen zu treffen, um die ziellose Schießerei sowohl der Kommandeure als auch der Mannschaft der Abteilung für immer auszumerzen und keinen einzigen Fall eines ziellosen Schießens im Standortbereich der Abteilung wie auch außerhalb dieses unbeachtet zu lassen.

Bevollmächtigter des Belorussischen Stabs

der Partisanenbewegung und des ZK der KP(b)

Belorußlands für das vereinigte Rajonzentrum Lida

(Sokolov)

F. 1329 , op. 1, d. 42,1.1.

\section{Dokument Nr. 2}

Befehl Nr. 0019 vom 2. Juni 1943 des Bevollmächtigten des ZK der KP(b)B und des Belorussischen Stabs der Partisanenbewegung für das vereinigte Rajonzentrum Ivenec über unkorrekte Beziehungen zwischen Abteilungen und Brigaden

Streng geheim

Expl. Nr....

$$
\text { Befebl }
$$

des Bevollmächtigten des $Z K$ der $K P(b) B$ und des Belorussischen Stabs der Partisanenbewegung für das vereinigte Rajonzentrum Ivenec
2. Juni 1943
Nr. 0019
Pušča N.

$\$ 1$

In den Brigaden und Abteilungen sind Fälle von unkorrekten, unkameradschaftlichen Beziehungen in der Mannschaft der Brigaden und Abteilungen zu beobachten. Anstatt kameradschaftlicher Geschlossenheit und gegenseitiger Hilfe im Kampf und in der Disziplin bestehen Antagonismen und Streitereien zwischen den Brigaden und Abteilungen. So haben die Partisanen der Frunze-Brigade das Marodieren und Rowdytum gegenüber der Zivilbevölkerung zugelassen und wälzen das auf die Partisanen der Stalin-Brigade ab, oft ist es auch umgekehrt, wenn die Partisanen der Stalin-Brigade oder einer anderen Brigade randalieren und das den Parti- 
sanen der Frunze-Brigade zuschreiben. Der Partisan Chmelevski aus der Dzeržinskij-Abteilung der Stalin-Brigade nahm einem Uhrmacher eine Uhr weg und stellte ihm eine Quittung im Namen der Lazo-Abteilung aus. Die Faschisten schleusen ihre Agenten zu dem Zweck ein, die Autorität der Partisanen in der Bevölkerung zu untergraben, und säen Feindschaft zwischen Abteilungen und Brigaden. Einige Kommandeure und Partisanen treffen keine Maßnahmen zur Beseitigung der vorhandenen Mängel, im Gegenteil, sie schüren sie noch mehr. Diese schändliche Erscheinung hat recht breite Ausmaße angenommen, oft wird dabei die Waffe als Argument angewandt. Zum Beispiel: Leutnant Ključnik, Kommandeur der Ščors-Abteilung, erschoß, ohne eine Beschuldigung zu erheben und ohne die Gründe zu erklären, den Kommissar der Lazo-Partisanenabteilung Zaskind und den Partisanen Petračkov. Der Kommissar und Gehilfe des Politruks der Dzeržinskij-Abteilung der Stalin-Brigade Ljachov erschoß drei Partisanen der Frunze-Brigade: Ražkov, Kurganov und Charčebnikov. Sergeant Anochin, Stabschef der Frunze-Brigade, erschoß Kavalev, den Kommissar und Partisanen der Budennyj-Abteilung. Der Zugführer der Stabskompanie der Stalin-Brigade Nekrasov führte im Dorf Kondratoviči eine Gruppe von Juden der Frunze-Brigade zur Erschießung. Man könnte noch Dutzende ähnliche Vorfälle anführen.

$\$ 2$

Es gibt Fälle des unerlaubten Übergangs von Partisanen von einer Abteilung zu einer anderen, einige Kommandeure fördern so etwas und werben Partisanen aus anderen Abteilungen an; so suchte der Kommandeur der „Bol’ševik“-Abteilung der Stalin-Brigade Evlamenko, als er sich im Dzeržinskij Rajon befand, Partisanen aus der Frunze-Brigade für seine eigene Abteilung zu gewinnen.

Ich befehle:

1. Den unerlaubten Erschießungen von Partisanen anderer Abteilungen und Brigaden ohne Sanktion der Untersuchungsorgane ein Ende zu setzen.

2. Ich verbiete es kategorisch, ohne offizielle Zustimmung Partisanen aus anderen Abteilungen aufzunehmen; die neu eintreffenden Partisanen aus anderen Abteilungen sind zu entwaffnen, und die Tatsache ist ihrem Dienstort zu melden.

3. Die Rolle des Kommandeurs ist zu erhöhen. Eine Verfügung des übergeordneten Kommandeurs ist für alle Partisanen, alle Abteilungen und Brigaden Gesetz.

4. Über die Aktionen der Partisanen in den Rajons, die den Abteilungen und Brigaden zugeteilt sind, ist eine Kontrolle einzuführen.

5. Der Antagonismus zwischen den Brigaden und Abteilungen sowie die grobe Behandlung der Zivilbevölkerung sind durch politische Aufklärungsarbeit in kürzester Zeit zu beseitigen.

6. Es ist jedermann strengstens verboten, als Vertreter anderer Brigaden und Abteilungen aufzutreten, die Verletzer sind streng zur Verantwortung zu ziehen.

7. Die Brigadekommandeure haben die Motive zu untersuchen, von denen sich die oben genannten Kommandeure und Partisanen bei den Erschießungen leiten ließen. Die Untersuchungsmaterialien sind mir spätestens am 20. Juni vorzulegen.

Der vorliegende Befehl ist jedem Partisanen zur Ausführung zur Kenntnis zu bringen.

Bevollmächtigter des $\mathrm{ZK}$ der $\mathrm{KP}(\mathrm{b}) \mathrm{B}$ und

des Belorussischen Stabs der Partisanenbewegung

für das vereinigte Rajonzentrum Ivenec

(Dubov) 
Stellvertreter des Bevollmächtigten des ZK der KP(b)B und

des Belorussischen Stabs der Partisanenbewegung

für das vereinigte Rajonzentrum Ivenec

und Leiter der operativen Abteilung

Major

(Vasilevič)

F. 1329, op. 1, d. 30, 11. 22-23

\title{
Dokument Nr. 3
}

\section{Befehl Nr. 0055 vom 16. Juni 1943 für die Stalin-Brigade über die Bekämpfung den Partisanen wesensfremder Handlungen}

Streng geheim

Expl. Nr. 8

\author{
Befebl \\ für die Partisanenbrigade „STALIN"
}

16. Juni 1943

Nr. 0055

Pušča N.

Als ich mit meinem Stellvertreter sowie mit Major Vasilevič, Stellvertreter des Bevollmächtigten des ZK der KP(b)B und des Belorussischen Stabs der Partisanenbewegung für das vereinigte Rajonzentrum Ivenec, die an die Pušča angrenzenden Dörfer besuchte, konnte ich feststellen, daß die Partisanenabteilungen, die den Namen des großen Stalin tragen, bei der Bevölkerung beliebt und berühmt sind. Das Volk demonstriert in allen Rajons seine aufrichtige Liebe zu den Partisanen der Stalin-Brigade. Diesen Ruhm haben unsere Partisanen und Partisaninnen in den Kämpfen für die Heimat gegen die faschistischen deutschen Okkupanten sowie dank korrekter Beziehungen zur Bevölkerung und der Sorge für diese verdient.

Wenn unsere Partisanen die Dörfer Beliza, Deražno, Nivnoe, die Rajons Voložin, Bakšty, Ivenec, Dzeržinsk u. a. besuchen, werden sie als die liebsten Gäste, als Retter vor dem verhaßten Feind und vor kleinen Räubergruppen aufgenommen. Die Bewohner des Dorfes Deražno sagten Major Vasilevič: „Wir werden die Partisanen der Stalin-Brigade verpflegen und einkleiden, sie sollen nur zu uns kommen. "Diese Worte des Volkes gereichen den Partisanen und Partisaninnen unserer Stalin-Brigade zur Ehre und machen sie stolz.

Die politische Öffentlichkeitsarbeit, die unlösbare Verbindung mit der Bevölkerung und gegenseitige Hilfe machen die Stärke der Partisanenbewegung aus. Die Partisanen und Partisaninnen sind Vertreter der Sowjetmacht im gegnerischen Hinterland, sie müssen absolut ehrliche Menschen sein. Das ganze Volk blickt voller Hoffnung auf die Partisanen, lauscht auf ihre Stimme und hört sie.

Doch finden sich einzelne Partisanen, die statt der flammenden Liebe zu ihrer Heimat eine antisowjetische Einstellung zur Bevölkerung zeigen und dadurch den erkämpften Ruhm und die wohlverdiente Liebe der Bevölkerung zu den Partisanen und Partisaninnen der Stalin-Brigade kompromittieren.

Die Partisanen der „Bol’ševik“-Abteilung Bojko und Maslov brachten ins Dorf 
Ugly bis zu 10-15 Liter Schnaps, Fleisch und andere Lebensmittel und ergaben sich eine Woche lang dem Saufen. Der Partisan Želesnjak aus der Dzeržinskij-Abteilung versuchte im Dorf Petriloviči eine Frau zu vergewaltigen, und die Partisanin Kostinkevič aus derselben Abteilung nahm im Dorf Borovoe der Bürgerin Olg'a Sovastjuk, die den Partisanen hilft, den ganzen Besitz weg. Der Zugführer Grybanov aus der Čapaev-Abteilung steckte in stark betrunkenem Zustand das Dorf Stajki in Brand. Der Zugführer der Stabskompanie Nekrasov ist sehr grob gegen die Bevölkerung. Der Politruk Samsonov aus der Suvorov-Abteilung, der im Mai einen Auftrag zu erfüllen hatte, ergab sich, statt den Auftrag rechtzeitig zu erfüllen, dem Suff und veranstaltete ziellose Schießereien in Ortschaften. Es gibt viele Fälle, wo die Partisanen die bei Bauern ausgeliehenen Pferde und Fuhren nicht zurückgeben. Man könnte viele solche Vorfälle nennen.

Außerdem sind in letzter Zeit auch mehrere Fälle aufgetreten, wo gegen meinen Befehl Nr. 0032 vom 24. April 1943 über die Aktionen der Abteilungen in den ihnen zugeteilten Rajons verstoßen wird. Die von den Partisanen und Partisaninnen in der Bevölkerung geleistete politische Erziehungsarbeit kann durch falsches Verhalten einiger Partisanen ohne weiteres zunichte gemacht werden.

Saufen, Marodieren, Plünderung und sonstige Vergehen gegen die Bevölkerung müssen als schwerstes Verbrechen gegen die Heimat gelten. Die Deutschen schleusen absichtlich ihre Agenten ein, einige von ihnen, die sich für Partisanen ausgeben, fügen der Bevölkerung viel Schaden zu und stoßen die Bevölkerung dadurch von den Partisanen ab.

Partisanen und Partisaninnen der mir anvertrauten Brigade! NIEMAND von Euch DARF VERGESSEN, daß Ihr den Namen eines Roten Partisanen der STALIN-Brigade tragt. Ihr seid der Heimat gegenüber verantwortlich, der Sinn Eures Lebens besteht darin, das Vertrauen des Volkes zu rechtfertigen. Euer Leben gehört dem Volk bis zum letzten Atemzug, bis zum letzten Tropfen Blut. Der Popularität und dem Ruhm, die die STALIN-Brigade genießt, muß jeder Partisan und jede Partisanin in Ehren und mit Stolz gerecht werden.

ICH BEFEHLE:

1. Die Kommandeure und Kommissare der Abteilungen, die Partei- und Komsomolorganisationen müssen die politische Erziehungsarbeit unter den Partisanen und Partisaninnen sowie unter der Bevölkerung der den Abteilungen zugeteilten Rajons verstärken.

2. Der Einstellung der Partisanen zur örtlichen Bevölkerung und umgekehrt muß große Beachtung geschenkt werden. Die Grobheit gegenüber der Zivilbevölkerung ist mithilfe von Aufklärungs- und politischer Arbeit in kürzester Zeit zu beseitigen.

3. In den Partisanenabteilungen ist absolute Ordnung zu schaffen; jene, die in ihrem Verhalten zur Bevölkerung gegen die Disziplin verstoßen, sind strenger zu behandeln. In allen Fällen, wo der Bevölkerung Schaden oder Kränkungen zugefügt wurden, sind strenge Maßnahmen zu treffen.

4. Ohne Notwendigkeit dürfen den Bauern die Pferde nicht weggenommen werden, und Pferde, die für eine Operation benötigt wurden, sind den Bauern rechtzeitig zurückzugeben.

5. Die Kommandeure und Kommissare der Abteilungen haben in Zusammenhang mit den von mir oben genannten Vorkommnissen in eigener Zuständigkeit 
gegen die Friedenstörer Disziplinarmaßnahmen zu treffen und mir bis zum 25. Juni Vollzug zu melden.

6. Es ist den Abteilungskommandeuren verboten, ohne meine Erlaubnis Operationen in den ihnen nicht zugeteilten Rajons durchzuführen.

7. Die Kommandeure und Kommissare der Abteilungen haben durchzusetzen, daß die Fragen der Disziplin, des Saufens und Marodierens in den Mittelpunkt der Arbeit der Partei- und Komsomolorganisationen gestellt werden.

Der Befehl ist mit der gesamten Mannschaft der Brigade durchzuarbeiten.

Brigadekommandeur Leutnant

Brigadekommissar

Stabschef der Brigade Oberleutnant
(Gulevič)

(Murašov)

(Karpov)

F. 1329 , op. 1, d. 35, 11. 23-24

\section{Dokument Nr. 4}

\section{Befehl Nr. 25 vom 21. Juli 1943 des Bevollmächtigten des ZK der KP(b)B und des Belorussischen Stabs der Partisanenbewegung für das vereinigte Rajonzentrum Ivenec über die falsche Einstellung zur örtlichen Bevölkerung}

\section{Befebl}

des Bevollmächtigten des $Z K$ der $K P(b) B$ und

des Belorussischen Stabs der Partisanenbewegung

für das vereinigte Rajonzentrum Ivenec

\section{Juli 1943}

Nr. 25

Pušča N.

Als ich Ortschaften im Raum der Operationen der Brigaden besuchte, hörte ich mehrere Beschwerden der Bevölkerung über das falsche Verhalten der Partisanen zur Bevölkerung. Diese Beschwerden sind in vielen Fällen gerechtfertigt und gerecht. Einige Kommandeure und Partisanen verstehen nicht, daß eine den Partisanen wesensfremde Einstellung zur Bevölkerung auf das strengste bestraft werden muß. Marodieren, Nötigung, Vergewaltigungen, Grobheit sind außerordentlich schwere Verbrechen. Das kann nur ein Werk der eingeschleusten deutschen Agenten sein, die sich als Partisanen ausgeben, der Bevölkerung viel Leid zufügen und so diese von den Partisanen abstoßen.

Die meisten Beschwerden werden gegen die Partisanen der Žukov-Brigade, die ehemalige Gruppe Juzik, die Gruppe Boženko und die Gruppe Šemetovec vorgebracht.

In den Ortschaften Naliboki, Nesteroviči, in Nivno, Deražno und Derevnoe gibt es mehrere Vergewaltigungsfälle. Schnaps wird fast immer durch angedrohte Erschießung erpreßt, es werden Pferde, die Kuh weggeführt, das Hab und Gut wird weggenommen. Die schändlichste Erscheinung ist die Verprügelung der Bevölkerung mit Ladestöcken, Gewehrkolben usw. Ein Beispiel aus dem Dorf Nivno: Die Partisanen aus der Abteilung des Kommandeurs Kurbanov versuchten, die Bürgerin Karpevič zu vergewaltigen und verprügelten ihre Kinder. In Deražno nahm der 
Partisan Parchimovič aus der Abteilung des Kommandeurs Kurbanov dem Bürger Vinevec den ganzen Besitz weg und versetzte ihm Brustschläge mit dem Gewehrkolben. Auf der Mühle Sutoki verlangte der Partisan Gus'kov (der Lahme) aus der Komsomolzenabteilung, der ohnehin schon betrunken war, unter Androhung der Erschießung noch mehr Schnaps. Am 12. Juni führte eine Gruppe von Partisanen der Čkalov-Brigade eine Hausdurchsuchung bei allen Bauern im Dorf Pil'nica durch. Die Aufzählung solcher Fakten ließe sich unendlich lange fortsetzen.

ICH BEFEHLE:

1. Partisanenkommandanturen in folgenden Ortschaften einzurichten:

a) Stalin-Brigade: in Nesteroviči, Naliboki, Terebejna, Nalibockaja Rudnja.

b) Čkalov-Brigade: in Rudnja Sivickaja, Borovikovšcizna, Jackovo, Rum.

c) Žukov-Brigade: in Zuborovo, Suporosnaja Sloboda, Derjažno, Nivno.

2. Die Kontrolle seitens der Brigaden über die Aktionen der Partisanen in den zugeteilten Rajons zu organisieren.

3. Alle Fälle einer den Partisanen wesensfremden Einstellung zur Bevölkerung zu untersuchen und gegen die Verletzer strenge Maßnahmen zu treffen.

4. Das ziellose Herumtreiben von einzelnen Männern durch die Dörfer zu verbieten. In den Lagern eine strenge Ordnung, Vollzähligkeitsappelle am Abend und Morgenappelle einzuführen. Personen, die sich zu einer Operation begeben, sind schriftliche Dienstaufträge auszustellen und von ihnen nach Ausführung vollständige Berichte mit allen kleinen Einzelheiten zu verlangen.

5. Ich verbiete, Verpflegungsoperationen in Ortschaften, die in einem Umkreis von $10 \mathrm{~km}$ an die Pušča angrenzen, und wirtschaftliche Beschaffungsoperationen in den anderen Brigaden zugeteilten Rajons durchzuführen.

6. Die Aufklärungsarbeit unter den Partisanen in Bezug auf ihr Verhalten gegenüber der Bevölkerung ist zu verstärken.

7. Ich verwarne den Kommandeur der Suvorov-Abteilung Kurbanov und den Kommandeur der Komsomolzenabteilung Kočanovič wegen der Zulassung von Marodieren, Prügeleien, Erpressung und Gewaltanwendung und fordere, daß sie solche Vorkommnisse unterbinden und nicht mehr zulassen.

8. Der Befehl ist jedem Partisanen zur Kenntnis zu bringen, auf Partei- und Komsomolzenversammlungen sowie auf den Beratungen des Kommandeurskorps durchzuarbeiten.

Bevollmächtigter des ZK der $\mathrm{KP}(\mathrm{b}) \mathrm{B}$ und

des Belorussischen Stabs der Partisanenbewegung

für das vereinigte Rajonzentrum Ivenec

(Dubov)

F. 1329 , op. 1, d. 30, 1. 29. 


\section{Dokument Nr. 5}

Internes Schreiben des Untergrund-Oblastkomitees des KP(b)B Baranoviči über den Kampf gegen Saufen und Marodieren.

(Sommer - Herbst 1943?)

Streng geheim

An die Sekretäre der Untergrund-Rajonkomitees der $K P(b) B$, die Kommandeure und Kommissare der Brigaden und Abteilungen der Oblast' Baranoviči

Internes Schreiben

Obwohl die Parteiorganisationen und die Kommandeure der Brigaden und Abteilungen eine große Arbeit geleistet haben, um Ordnung zu schaffen und Fälle von Marodieren und Saufen zu beseitigen, kommt es in den Abteilungen und Brigaden immer noch zu unzulässigen Operationen einzelner Partisanen, die den hohen $\mathrm{Na}$ men des Sowjetpartisanen kompromittieren und dadurch die Partisanenbewegung unterminieren. Die durchgeführte Überprüfung hat festgestellt:

1. Im Ort Rubeževiči verlangte der Partisan Ivan Meleško aus der DzeržinskijBrigade in betrunkenem Zustand von einem Bauern Feuersteine für sein Feuerzeug und drohte, ihn sonst zu erschießen.

2. Im Dorf Pil'nica riß ein gewisser N. Kudlič aus der Abteilung „Bol'ševik“ in betrunkenem Zustand dem Bürger Lipnickij dessen letzte Jacke vom Leib und verprügelte ihn.

3. Beim Aufenthalt von zwei Partisanen aus der Suvorov-Abteilung der FrunzeBrigade auf dem Gehöft Serkulevskie verlangte einer von ihnen, Arkadij Levkovič, vom Bürger Jossif Skurat zwei Liter Schnaps. Als ihm geantwortet wurde, daß es keinen Schnaps gebe, durchsuchte er das Haus. Levkovič fand keinen Schnaps, dafür aber einen Pelzkragen von einem Damenmantel und nahm ihn an sich. Der Hausherr wurde gezwungen, um den Schnaps zu den Nachbarn zu gehen. Als Levkovič den Schnaps bekam, gab er den Kragen zurück.

4. Der Partisan Mickevič aus der Frunze-Brigade zerstörte auf dem Gehöft Mikuliči 4 Bienenstöcke des Bürgers Iosif Dovnar. Ebenfalls dort verprügelte der Kommandeur der Kutuzov-Abteilung Golicev in betrunkenem Zustand ohne jeden Grund den Bürger Julijan Jankovskij.

5. Eine Gruppe von Partisanen aus der Dzeržinskij-Brigade beschaffte Roggen im Dorf Teleševiči. Als die Bauern ihnen das Getreide brachten, verprügelte der Partisan Rjabcev, der betrunken war, die Bäuerin Lidija Bondak, weil sie zu wenig mitgebracht habe.

6. Ein Partisan der Abteilung „Bol'ševik“ riß in Rubeževiči zusammen mit Šipulin (der ebenfalls betrunken war) dem Bauern Fedor Domejko die Kleidung vom Leib und drückte ihm den Hals zu.

7. Der Leiter der Aufklärung des Stalin-Stabs belästigte in betrunkenem Zustand ein junges Mädchen im Dorf Mešiči, bis sie weinend vor ihm floh.

8. Der ehemalige Polizist Mintjuk, heute Partisan der Abteilung „Für Sowjet-Belorußland", terrorisiert die Bevölkerung systematisch. Vor kurzem verprügelte er eine Bäuerin, der er Leinen wegnahm, um die Wände seiner Erdhütte damit zu beziehen. Zuvor hatten die Deutschen das Haus dieser Frau in Brand gesteckt. 
9. Es kommt vor, daß die Partisanen unnötigerweise den Bauern die Pferde wegnehmen und nicht zurückgeben. Sie nehmen auch Kleidungsstücke weg, die die Partisanen gar nicht brauchen. Es gibt Fälle, wo die weggenommenen Pferde und Kleidungsstücke gegen Schnaps getauscht werden.

10. Der Partisan Konstantin Deniskevič aus der Budennyj-Abteilung verlangte vom Bürger Spičenko im Dorf Ponižany Schnaps und begann eine Schießerei. In einer Reihe von Abteilungen und Brigaden wird zugelassen, daß kleine Gruppen in den Rajon fahren, und in einigen Fällen ergeben sie sich dem sinnlosen Saufen in den Dörfern und behandeln die Bevölkerung rücksichtslos. Einzelne Kommandeure und Kommissare haben ihre Verantwortung vor der Partei und der Heimat vergessen, haben vergessen, $\mathrm{da} ß$ sie im gegnerischen Hinterland die Sowjetmacht vertreten, und statt gegen die korrupten Elemente, Gewalttäter und Marodeure durchzugreifen, beziehen sie die Position von „Beobachtern“ und behaupten: Bei uns hat es so was nicht gegeben, kann es das gar nicht geben, usw.

1) Das Baranovičier Untergrundkomitee Belorußlands betrachtet diese Erscheinung als unerträglich und verlangt von allen Untergrund-Rajonkomitees der $\mathrm{KP}(\mathrm{b}) \mathrm{B}$, allen Kommandeuren und Kommissaren der Partisanenbrigaden und -abteilungen, von allen Kommunisten und Komsomolzen eine entschlossene Verstärkung des Kampfes gegen die Erscheinungen wie Marodieren, Saufgelagen, Rowdytum und sonstige Äußerungen einer unkorrekten, dem Sowjetpartisanen wesensfremden Einstellung zu unserer Bevölkerung. Es gilt, eine Atmosphäre der Unduldsamkeit gegenüber all jenen (ohne Ansehen der Person) zu schaffen, die an den oben genannten Vorkommnissen schuld sind oder sie zulassen. Jeder Fall von Marodieren, Forderungen nach Schnaps bei den Bauern, Prügeleien, Durchsuchungen u.a. muß von allen Partisanen erörtert und die Schuldigen müssen bestraft werden als freiwillige oder zufällige Feinde, die durch ihre Handlungen den deutschen Besatzern helfen. Gegen sie müssen harte Strafmaßnahmen bis hin zur Erschießung angewandt werden.

2) Es ist notwendig, die politische Erziehungsarbeit unter den Partisanen und in der Bevölkerung radikal zu verbessern, denn eine Reihe von Rajonparteikomitees und Partei- und Komsomolorganisationen sowie der politische Apparat der Abteilungen und Brigaden haben diese Arbeit vernachlässigt.

Das Oblastkomitee verpflichtet die Kommissare der Brigaden und die Rajonparteikomitees, zweimal im Monat dem Oblastkomitee der $\mathrm{KP}(\mathrm{b}) \mathrm{B}$ in speziellen politischen Meldungen Rechenschaft über die geleistete Arbeit abzulegen.

Das Oblastkomitee der $\mathrm{KP}(\mathrm{b}) \mathrm{B}$ verpflichtet die Rajonkomitees der $\mathrm{KP}(\mathrm{b}) \mathrm{B}$, gemeinsam mit den Kommandeuren und Kommissaren der Brigaden und Abteilungen jeder Brigade Zonen (mit ausführlicher Aufzählung der Dörfer) zuzuteilen, in denen sie politische Arbeit zu leisten haben und Verpflegung beschaffen können.

Die Rajonkomitees müssen sich in jedem Rajon, ausgehend von der Versorgungslage und anderen Besonderheiten, an die Normen halten, nach denen die Dorfbauern die Verpflegung liefern sollen.

Das Oblastkomitee empfiehlt, für jeden Bauernhof bzw. jedes Dorf je nach den örtlichen Bedingungen Normen der Lieferung von Getreide, Fleisch und anderen Lebensmitteln für die Partisanen festzulegen und nicht zuzulassen, daß Lebensmittel in nicht organisierter Form weggenommen werden.

Die Einführung eines solchen Verfahrens wird es, da die Bauern den Partisanen 
gern helfen werden, ermöglichen, die Verpflegung schnell zu beschaffen und Fälle des Marodierens und ziellosen Herumtreibens von Partisanengruppen in den Dörfern zu verhindern.

3) Verboten wird die Entsendung von kleinen Gruppen zu Verpflegungsoperationen; zu empfehlen ist die Entsendung mindestens eines Zuges mit dem Zugführer, einem Kompaniechef oder einem Politruk an der Spitze, wobei der jeweilige Kommandeur die volle Verantwortung für die Handlungen seiner Einheit trägt.

4) Das Sowjetvolk umgibt die Volksrächer, die Partisanen, mit Sorge und Aufmerksamkeit, weil es in ihnen seine besten Freunde und Verteidiger gegen die deutsche Willkür sieht. Wir müssen auf diese Unterstützung und Liebe des Volkes stolz sein und sie schätzen, denn darin liegt die Quelle unserer Stärke. Das Oblastkomitee der KP(b)B bringt die feste Überzeugung zum Ausdruck, daß die Partei- und Komsomolorganisationen, die Kommandeure der Brigaden und Abteilungen bolschewistische Ordnung schaffen und die Fälle von Marodieren ausmerzen, die politische Arbeit verstärken und die Beschlüsse des 5. Plenums des $\mathrm{ZK}$ der $\mathrm{KP}(\mathrm{b})$ Belorußlands voll und ganz erfüllen werden.

5) Dieses Schreiben ist in den Partei- und Komsomolzenversammlungen der Brigaden und Abteilungen zu erörtern. Die Rajonkomitees der $\mathrm{KP}(\mathrm{b}) \mathrm{B}$ haben Beratungen des politischen Kommandeurskörpers über diese Fragen abzuhalten. Mit den Partisanen sind spezielle Aufklärungsgespräche zu führen.

Sekretär des Untergrund-Oblastkomitees

Baranoviči der $\mathrm{KP}(\mathrm{b}) \mathrm{B}$

(Platon)

$\mathrm{Zu}$ verschicken nur an die Rajonkomitees der $\mathrm{KP}(\mathrm{b}) \mathrm{B}$.

Nach Durchführung der Versammlungen und Beratungen

ist das Dokument durch Verbrennen zu vernichten.

F. 1329, op. 1, d. 29, 11. 130-132.

\section{Dokument Nr. 6}

Politmeldung A. Gajsarovs, Kommissar der Žukov-Brigade, über den politischmoralischen Zustand seiner Einheit (15. Dezember 1943 bis 1. Januar 1944)

Streng geheim

Dem Bevollmächtigten des $Z K \operatorname{der} K P(b) B$ und

des Belorussischen Stabs der Partisanenbervegung

für die Oblast' Baranoviči

Generalmajor Gen. Platon

Kopie: an Gen. Carjuk

Vom Kommissar der Brigade

„Marschall der UdSSR Žukov“

Politmeldung über den politisch-moralischen Zustand

Der Mannschaften der Abteilungen

der Brigade "Marschall der UdSSR Žukov"

vom 15. Dezember 1943 bis 1. 1. 1944 
Bei der Gestaltung der politischen Massen- und Erziehungsarbeit galt die Aufmerksamkeit vor allem der Notwendigkeit, die Weisungen des internen Schreibens des Oblastkomitees der KP(b)B Baranoviči zu befolgen, um gute Beziehungen zur Bevölkerung aufrechtzuerhalten und Fälle von Saufen und Marodieren auszurotten.

Folgende Arbeit wurde geleistet:

Am 18. Dezember 1943 wurde eine Beratung der Kommandeure und Politkader der Brigade durchgeführt, die das interne Schreiben des Oblastkomitees erörterte. In den Abteilungen der Brigade fanden Partei- und Komsomolzenversammlungen, Versammlungen der Mannschaften, auch Beratungen der Unterführer und aller Kommandeure der Abteilungen statt. Den Abteilungen wurden Dörfer zugeteilt, in denen sie die Beschaffung organisiert durchführen und die Erziehungsarbeit in den Massen leisten sollen. Alle Abteilungen leisten eine breite Öffentlichkeitsarbeit. Versammlungen und Gespräche mit der Bevölkerung wurden durchgeführt, bei denen es um die Gestaltung guter gegenseitiger Beziehungen und die Notwendigkeit ging, den Partisanen mehr zu helfen. Die Bevölkerung nahm die konkreten Vorschläge und Maßnahmen der Partisanenabteilungen mit großer Begeisterung auf.

Die Voronov-Abteilung veranstaltete zwischen dem 15. Dezember 1943 und dem 1. Januar 1944 acht Gespräche mit der Bevölkerung, an denen sich 445 Personen beteiligten. Es waren 17 Kommunisten, Komsomolzen und Parteilose, gut vorbereitete Agitatoren, die die Gespräche durchführten.

Um den Abteilungen der Brigade bei einer besseren Realisierung der vorgemerkten Maßnahmen zu helfen, unternahmen die Kommandeure der Brigade Fahrten in den Operationsraum der Suvorov-, der „Mstitel'“- und der Rokossovskij-Abteilung. Wir haben persönlich Versammlungen und Gespräche mit der Bevölkerung in den Dörfern Atalez', Borok, Žukov-Borok u. a. durchgeführt.

Die Kommandeure und Mannschaften der Abteilungen haben die vor ihnen stehenden Aufgaben, gute gegenseitige Beziehungen zur Bevölkerung herzustellen, verstanden. Im Ergebnis der umfangreichen Arbeit unter den Mannschaften und in der Bevölkerung ist die Zahl der Fälle von Saufgelagen, des Marodierens und der unsowjetischen Einstellung zur Bevölkerung stark zurückgegangen.

Die Abteilungen unternehmen ihre Fahrten in den Rajon in organisierter Weise, die Suvorov- und die „Mstitel'“-Abteilung halten sich ständig in ihren Rajons auf und führen die Beschaffung von Lebensmitteln organisiert durch, veranstalten Hinterhalte und stören den gegnerischen Nachrichtenverkehr.

Trotz der mit den Mannschaften geleisteten großen Arbeit gibt es noch Fälle unsowjetischer Einstellung zur Bevölkerung und andere Verstöße. So haben die Partisanen Konstantin Lopko und Ivan Ston'ko aus der Suvorov-Abteilung im Dorf Semenčicy einen Bürger verprügelt, um von ihm Waffen zu bekommen. Wegen dieser Vergehen wurden sie zu 10 Tagen verschärfter Haft verurteilt. Die Partisanin Marusja Senina schlug im Dorf Zukov-Borok eine Frau, weil sie ein Kleid von ihr wollte; deswegen wurde Senina zu 4 Tagen verschärfter Haft verurteilt. Der Partisan Vasilij Černov ergab sich im Dorf [...] dem Suff, weshalb er für 5 Tage verhaftet wurde.

Am 22. Dezember 1943 brachten die Partisanen Monič und Kljekij aus der Rokossovskij-Abteilung bei der Rückkehr von einer Verpflegungsoperation dem Bürger Jankovskij, der in der Pušča lebt, 3 kg Salz im Tausch gegen Schnaps; dẹswegen wurden ihnen vier Tage Zusatzdienst auferlegt, und Jankovskij wurde verwarnt. 
Am 22. Dezember 1943 erpreßte der Partisan Lopato aus dem Kommandantzug des Brigadestabs den Bürger Jozef Loman aus dem Dorf Borok, um Schnaps von ihm zu bekommen, weswegen Lopato disziplinarisch bestraft wurde.

Es kommt vor, daß Partisanen des vereinigten Rajonzentrums Ivenec Streifzüge in den Operationsraum unserer Brigade unternehmen. Am 24. Dezember 1943 beispielsweise kamen 27 Partisanen aus der Abteilung von Zorin zur Beschaffung ins Dorf Borok, wobei sie die entsprechende Erlaubnis von Gen. Dubov hatten. Außerdem liegen viele Beschwerden der örtlichen Bevölkerung über die Aktionen von Minin vor, der in unserem Rajon Verpflegungsoperationen durchführt, wobei er die Bevölkerung buchstäblich terrorisiert. Zu dieser Frage schicken wir Ihnen eine spezielle ausführliche schriftliche Meldung, damit Sie ihn zur Verantwortung ziehen.

Am 2.-4. Januar 1944 hielt ich mich im Raum der Ortschaft Derevnoe auf. Viele Bauern beschweren sich über die Partisanen der Čapaev-Abteilung, die groben Unfug treiben. Ein Beispiel: Am 3. Januar 1944 trafen in der Ortschaft Derevnaja nachts Partisanen aus der Čapaev-Abteilung ein, gingen durch die Häuser, veranstalteten Durchsuchungen und ließen alles mitgehen, was ihnen unter die Augen kam. Beim Bürger Aleksandr Kolovčut veranstalteten sie eine totale Durchsuchung, nahmen ihm zwei Bettdecken, Kinderwindeln, Gesichtspuder, 3 Hemden, eine Unterhose, ein Kummet, den Pferdezaum und den ganzen Machorkavorrat weg und bedrohten ihn schwer, damit er ihnen seine polnischen Offiziersstiefel gab. Als sie Fotos entdeckten, verlangten sie die Fotokamera, was sie damit begründeten, daß es eine geben müsse, wenn Fotos da seien.

Dem Bürger Pavel Romanovič wurde das kranke Pferd weggenommen, das der Partisan I. I. Mamlaj aus der Kalinin-Abteilung der Komsomolzenbrigade zur Behandlung dagelassen hatte. Die vorgewiesene Bescheinigung Mamlajs über das Pferd nahmen die Partisanen an sich und führten das Pferd weg.

Am 28. Dezember 1943 betranken sich der Zugführer Čekin aus der VoronovAbteilung, der Kommandeur der Unterabteilung Semin und der Partisan Karapuzov in ihrer Abteilung, machten die Partisanin Lida Orechova betrunken, versprachen ihr, sie ins Familienlager zu bringen, wo sie mit ihrem einmonatigen Kind lebt, und vergewaltigten sie unterwegs. Über diesen Fall ist eine Untersuchung eingeleitet worden.

Unter den Kommunisten gibt es keine Störenfriede, unter den Komsomolzen wurden zwei Fälle festgestellt.

In den Abteilungen der Brigade fanden Festsitzungen zum 25. Jahrestag der BSSR statt, aus demselben Anlaß wurden auch Partei- und Komsomolzenversammlungen, Gespräche unter den Mannschaften durchgeführt und Jubiläums-Wandzeitungen herausgegeben. Versammlungen und Gespräche anläßlich des 25. Jahrestags der BSSR wurden auch in den Dörfern veranstaltet.

In allen Abteilungen wurde der Kampfrapport des Oblastparteikomitees an die Regierung der BSSR und das ZK der KP(b)B angenommen.

Vorkommnisse:

Im betreffenden Zeitraum sind 7 Partisanen gefallen, wurden 3 Vaterlandsverräter erschossen, es gab einen Deserteur. Unter den Gefallenen ist der Kommandeur der Sprengpioniergruppe der Suvorov-Abteilung Nikolaj Timofeevič Fofonov, er wurde am 19.12. 1943 bei der Erfüllung einer Gefechtsaufgabe getötet. Gen. Fofonov war Kandidat der KPdSU(b). 
Am 27. 12. 43 wurden der Aufklärer der Suvorov-Abteilung K. K. Kivovič und der Partisan F. Dvorjankin auf dem Gehöft beim Dorf Borok von der StarinskajaPolizei umzingelt und getötet.

Der Partisan der Abteilung „Mstitel”“ M. S. Pitkevič wurde am 20. Dezember 1943 im Dorf Opečka von den Deutschen getötet.

Am 27. 12. 43 wurde der Partisan der Abteilung „Mstitel’“ I. A. Lapko im Dorf Borok von der Starinskaja-Polizei getötet.

Am 31. Dezember 1943 wurden G. P. Vasil'ev und S. M. Rajskij aus der Abteilung „Mstitel'“ von der Starinskaja-Polizei im Dorf Šeški getötet.

Aus der Rokossovskij-Abteilung desertierte am 19. Dezember 1943 der Partisan Vladimir Ivanovič Pustochod, der sich unter Mitnahme eines Revolvers ostwärts absetzte.

Der Obersergeant Ivan Ivanovič Vel'činskij und der Sergeant Bronislav Pavlovič Govorskij aus der Rokossovskij-Abteilung wurden vom Leiter der Sonderabteilung der Brigade als Vaterlandsverräter erschossen; beide kamen im November 1943 in die Abteilung (aus Minsk), erwiesen sich jedoch als deutsche Spione, ergaben sich systematisch dem Suff und terrorisierten die Bevölkerung.

Am 20.12. 43 betrank sich Vel'činskij und drohte, den Abteilungskommandeur Gen. Kačanovskij zu erstechen.

Am 24. Dezember 1943 waren Vel'činskij und Govorskij im Dorf Berežno, betranken sich und warben gewaltsam junge Leute zu den Partisanen an; einigen schlugen sie vor, sich mit Schnaps vom Beitritt zu den Partisanen loszukaufen.

Gen. Stepčenko erteilte dem Sergeanten der Staatssicherheit Michail Nikiforovič Metel'skij (Kommandanturzug der Brigade) einen Sonderauftrag. Metel'skij hatte Angst vor Schwierigkeiten, die mit der Ausführung des Auftrags verbunden waren, desertierte, wurde in Senjawskaja Sloboda gestellt und als Vaterlandsverräter erschossen.

Kommissar der Brigade

„Marschall der UdSSR Žukov“

Major

(Gajsarov)

F. 1329, op. 1, d. 69, 11. 24-26.

\section{Dokument Nr. 7}

Befehl Nr. 2 vom 14. Januar 1944 des Bevollmächtigten des ZK der KP(b)B und des Belorussischen Stabs der Partisanenbewegung für das vereinigte Rajonzentrum Ščučinskij, Oblast' Baranoviči, über den Kampf gegen das Saufen und Marodieren

Streng gebeim

Befehl

des Bevollmächtigten des $Z K \operatorname{der} K P(b) B$ und

des Belorussischen Stabs der Partisanenbewegung

für das vereinigte Rajonzentrum Ščučinskij,

Oblast' Baranoviči 
Obwohl beim erwähnten vereinigten Rajonzentrum Ščučinskij eine Beratung mit den Kommandeuren und Politoffizieren der Abteilungen und Brigaden durchgeführt und den Einheiten das interne Schreiben des Oblastzentrums zugeschickt wurde, worin die Rede davon war, Ordnung zu schaffen und Fälle von Marodieren und Saufen unter einzelnen korrupten Partisanen zu beseitigen, sind bis jetzt in den Abteilungen und Brigaden immer noch unzulässige Handlungen einiger Partisanen zu verzeichnen, die den ehrenvollen Namen des Sowjetpartisanen kompromittieren und dadurch unsere Partisanenbewegung unterminieren, was die große Gefahr in sich birgt, in der örtlichen Bevölkerung Opposition gegen uns hervorzurufen.

Bei der durchgeführten Überprüfung wurde festgestellt:

1. Der Partisan A. Andrijanov aus der Vorošilov-Brigade verlangte von einem Bewohner des Dorfes Ust'e Schnaps, verprügelte ihn und schlug ein Fenster ein, und als er den Schnaps bekam, betrank er sich und schlief ein.

2. Der stellvertretende Kommissar der Vorošilov-Brigade Bilej und der Partisan derselben Brigade Drjabin verlangten vom Bürger Koska gesetzwidrig Schnaps und drohten ihm dabei mit der Erschießung.

3. Der Partisan V. Bobrin aus der Abteilung „Bor’ba“ (Kampf) der Lenin-Partisanenbrigade verlangte bei einem Bewohner des Dorfes Kolminskie Schnaps und drohte ihm, sonst das Pferd wegzunehmen.

4. Der Partisan Ivanov aus der Abteilung „Nepobedimyj“ der Lenin-Brigade kam ins Dorf Chodeviči und verlangte bei den Bauern Schnaps, worauf er ins Haus des Bürgers Moissej Balja ging und sich dort betrank, so daß die Polizei die Möglichkeit erhielt, das Haus einzukreisen, und Ivanov nur dank einem glücklichen $\mathrm{Zu}$ fall entkommen konnte.

5. Der Partisan Viktor Kordas aus der Abteilung „Bor’ba“ nahm im Dorf Kročli dem Bürger Jurčelevič (Partisanenfamilie) die letzte Kuh weg. Der Kommandeur der Abteilung „Bor'ba“" weiß davon, hat jedoch keine Maßnahmen getroffen.

6. Der Partisan Grincevič aus der Abteilung „Bor'ba“ und der Partisan Misarevič aus der Abteilung „Zvezda“ (Kommandeur: Kazakov) schlugen zwei Bienenhäuser des Bürgers Ivan Ivanovič aus dem Dorf Začeniči kaputt und nahmen den Honig mit, so daß die Bienen eingingen.

7. Der Kommissar des Brigadelazaretts Ivan Kalmikov verlangte vom Lazarettleiter Mehl, um Selbstgebrannten daraus herzustellen, und als ihm das verweigert wurde, packte er den Lazarettleiter Doktor Mjasnik am Hals, würgte ihn und drohte ihm mit der Erschießung.

8. Die Partisanen Grigorij Konoplev und A. Okulevič aus der Abteilung „Krasnogvardejskj" rissen im Dorf Korsački aus fünf Bienenstöcken der Bürger Ivan und Valerian Venskij den ganzen Honig heraus, so daß alle Bienen eingingen, dann plünderten sie im selben Dorf eine Flüchtlingsfamilie aus.

9. Die Partisanen Ušakov und Nazarov aus der Abteilung „Bor’ba“ schlugen im Dorf Korytnica in betrunkenem Zustand mit Ladestöcken auf die Bürger Grigorij Korda, Petr Ignat'evič Lun', Petr Ivanovič Lun' und andere ein, dann verlangten sie beim Bürger Kirel' anderthalb Liter Schnaps, und als der letztere nur einen halben Liter brachte, stahlen sie alle Sachen und veranstalteten eine Schießerei in seinem Haus.

10. Die Partisanen Šejbak und ein zweiter, genannt „Usbeke“, aus der Lenin-Ab- 
teilung verlangten im Dorf Gnanskie Schnaps, und als die Hausfrau Melja ihnen keinen gab, stellten sie die Familie zur Erschießung auf. Die Familie blieb nur durch einen Zufall am Leben, weil in dieser Zeit Partisanen aus einer anderen Abteilung im Dorf eintrafen.

11. Der Partisan Ivančuk aus der Brigade „Pobeda“ (Sieg) entfernte sich unerlaubterweise aus dem Lager, betrank sich im Dorf Ostrovo und verprügelte in diesem Zustand einen Bewohner dieses Dorfes.

Einzelne Kommandeure und Kommissare haben gegen die außer Rand und Band geratenen Marodeure und Säufer keine entschlossenen Maßnahmen getroffen, ganz im Gegenteil: Sie waren bemüht, den wirklichen Sachverhalt zu verheimlichen, beschränkten sich auf Gespräche und bewogen dadurch die korrupten Elemente dazu, ihre schändlichen Handlungen gegenüber der örtlichen Bevölkerung fortzusetzen.

Zwecks Beseitigung der oben genannten Fälle von Marodieren, Saufgelagen und Verprügelung der Bevölkerung befehle ich:

1. Die Kommandeure und Kommissare der Brigaden und Abteilungen müssen entschlossen gegen die Erscheinungen von Marodieren, Suff und sonstigen den Partisanen wesensfremden Handlungen kämpfen, und zwar durch eine breit angelegte Überzeugungsarbeit in den Mannschaften, ohne auch nur einen Fall ungestraft zu lassen. Auf besonders böswillige Marodeure, Säufer und Gewalttäter sind die härtesten Strafen bis hin zur Erschießung anzuwenden.

2. Die Entsendung kleiner Gruppen zur Verpflegungsbeschaffung ist zu verbieten, zu diesem Zweck ist zumindest ein Zug mit dem Zugführer oder einem Kompaniechef zu entsenden.

3. Der Zugführer oder der Kompaniechef trägt die volle Verantwortung für die Handlungen seiner Einheit.

4. Die Brigadekommandeure verpflichte ich, in der Regel periodisch Gruppen von mindestens 50 Personen in die Zonen ihrer Handlungen zu entsenden, wo sie auftauchende Gruppen des Gegners zu vernichten und zugleich eine breite Aufklärungsarbeit in der Bevölkerung zu leisten sowie den dem Partisanengeist fremden Handlungen einzelner Partisanen, die ziellos durch die Dörfer ziehen, einen Riegel vorzuschieben haben.

5. Ich verpflichte alle Brigade- und Abteilungskommandeure, die in Ortschaften einzelne Partisanen ohne schriftlichen Auftrag feststellen, sie zu entwaffnen und in die entsprechende Einheit zu befördern; deshalb ist die Entsendung von Partisanen in die Ortschaften ohne Anweisung zu verbieten.

Ich verpflichte die Brigadekommandeure, am 15. und 30. jedes Monats mir über Fälle von Marodieren, Saufen und anderer den Partisanen wesensfremder Handlungen und die dazu getroffenen Maßnahmen Meldung zu erstatten, wobei besonders böswillige Fälle mir unverzüglich zu melden sind.

Bevollmächtigter des $\mathrm{ZK}$ der $\mathrm{KP}(\mathrm{b}) \mathrm{B}$ und des Belorussischen Stabs der Partisanenbewegung für das vereinigte Rajonzentrum Ščučinskij, Oblast' Baranoviči

(Šupenja)

F. 1329, op. 1, d. 82, 11. 51-52. 


\section{Dokument Nr. 8}

\section{Schriftlicher Bericht des Leiters der Sonderabteilung der Stalin-Brigade vom 19. Januar 1944}

\section{Streng geheim}

An Oberstleutnant G. B. Donskoj

Kopie: An den Sekretär des Rajonkomitees Ivenec der KP(b)B Gen. Dubov

Im Dorf Pil'nica, Rajon Ivenec, lebt eine gewisse Ljubov Petrovna P., geb. 1925, Einwohnerin der Stadt Minsk. Wahrscheinlich war die Bürgerin P. Partisanin in einer Abteilung der Dzeržinskij-Brigade und wurde aus dieser vertrieben. In Pil'nica hält sich die Bürgerin P. seit mehr als 2 Monaten auf, hat einen unmoralischen Lebenswandel und verkehrt geschlechtlich mit Partisanen der Abteilungen, die das Dorf Pil'nica passieren.

In meinem Auftrag untersuchte der Militärarzt dritten Ranges der Abteilung „Bol'ševik“ Gen. Lifšic am 17.1. 1944 die P. und stellte fest, daß sie in höchstem Maße verdächtig ist, Tripper zu haben.

Ich bitte, die Leitung der Dzeržinskij-Brigade zu veranlassen, daß sie die Bürgerin P. aus dem Dorf Pil'nica entfernt. Wenn die Leitung der Dzeržinskij-Brigade die entsprechenden Maßnahmen nicht trifft, werde ich gezwungen sein, meinerseits Maßnahmen gegen die Bürgerin P. als Ansteckungsträgerin einer Geschlechtskrankheit zu treffen.

Zugleich habe ich Ihnen einen anderen empörenden Fall zu melden: Die Partisanen der Dzeržinskij-Brigade Judin und Trusov erniedrigten einen Sowjetbürger.

Vor einigen Monaten wurde eine Gruppe von Sowjetmenschen aus Minsk herausgeführt, darunter Sofija Aleksandrovna Garbuz, geb. 1923, ihr Vetter Nikolaj Michailovič Mironenko und zwei weitere Genossen aus dieser Gruppe. Aus Minsk führte sie die Verbindungspartisanin Katja heraus. Als sie im Dorf Śpil'ki, Dzeržinskij Rajon, angekommen waren, wurden die Männer mit Gen. Nikolaj Michailovič Mironenko an der Spitze in eine Abteilung der Dzeržinskij-Brigade entsandt, während Judin und Trusov Sofija Garbuz angeblich als Köchin bei sich leben ließen. Etwas später brachten sie die Bürgerin Garbuz zum Tonovskie-Gehöft, Rajon Ivenec, und ließen sie dort allein. Mehr als zwei Monate mußte sie in diesem Gehöft buchstäblich betteln. Einmal kam der Partisan Trusov zu ihr und wollte sie zur Frau nehmen, aber sie weigerte sich, und er fuhr weg. Am 13.1.1944 ist Sofija Garbuz ins Dorf Pil'nica gekommen, wartet darauf, daß jemand aus der Dzeržinskij-Brigade sie abholt, und lebt weiter in Elend.

Über die Bürgerin Sofija Garbuz wurde folgendes bestätigt: Ihr Bruder Il'ja Aleksandrovič Garbuz, geb. 1915, gehört zu einer Abteilung der Vorošilov-Brigade. Ihre Vetter Ivan Dmitrevič Anan'ko und Sergej Nikolaevič Anan'ko sind Partisanen in der Ponomarenko-Brigade, der dritte Vetter Nikolaj Michailovič Mironenko gehört zur Dzeržinskij-Brigade.

Mitteilung für Ihre Reaktion.

Leiter der Sonderabteilung der Stalin-Brigade

19. Januar 1944

(Parafenjuk)

F. 1329 , op. 1, d. 33, 1. 7 . 


\section{Dokument Nr. 9}

Befehl Nr. 52 vom 5. Februar 1944 der Leitung der Žukov-Brigade über die Saufgelage des Stabschefs der Abteilung „Mstitel'“ der Žukov-Brigade

An Gen. Carjuk

Befehl

für die Partisanenbrigade „Marschall der UdSSR Gen. Žukov"

5. Februar 1944

Nr. 52

Pušča N.

Anhand der von der Sonderabteilung der Partisanenabteilung „Mstitel'“ der Žukov-Brigade durchgeführten Untersuchung des Falls stelle ich fest:

Am 28. Januar fuhr eine Gruppe von Partisanen der Abteilung „Mstitel”“ unter dem Befehl des Stabschefs der Abteilung, Ivan Pantelejevič Lemeš, in den Rajon Stolbcy, um einen Kampfauftrag zu auszuführen.

Unterwegs machte die Partisanengruppe einen kurzen Halt im Dorf Sloboda. Anstatt sich von dort zur Erfüllung des Kampfauftrags zu begeben, betrank sich der Stabschef Lemeš, so daß er seine Unterstellten nicht leiten konnte und die Erfüllung des Auftrags vereitelte.

In der Nacht vom 28. zum 29. Januar 1944 hätte die Gruppe sich zur Erfüllung des zweiten Kampfauftrages begeben sollen, aber auch in dieser Zeit ergab sich Lemeš dem Saufen. Als der Partisan Kul'ša ihn an seine Pflicht erinnerte, entwaffnete ihn Lemeš, verprügelte ihn und drohte ihm mit der Erschießung. Wegen Lemeš' Saufgelagen wurde der Auftrag nicht erfüllt.

Der Stabschef der Abteilung „Mstitel'“ verstieß somit gegen den Befehl Nr. 40 mit dem Verbot des Branntweinbrennens und des Saufens.

Er verging sich grob gegen die Partisanenehre und Bürgerwürde des Partisanen Kul'ša.

Aufgrund des oben Dargelegten befehle ich:

den Stabschef der Partisanenabteilung „Mstitel'“ Ivan Pantelejevič Lemeš von seinem Posten abzusetzen.

Ich stelle den Antrag, daß die übergeordneten Organe I. P. Lemeš den Dienstgrad aberkennen und ihn in die Verfügungsgewalt des Brigadestabs übergeben.

Kommandeur der Žukov-Brigade

Oberst

Kommissar der Žukov-Brigade

Major

Stabschef der Žukov-Brigade

Hauptmann

(Vasilevič)

(Gajsarov)

(Kajdalov)

F. 1329, op. 1, d. 68, 1. 149 . 


\title{
Dokument Nr. 10
}

\section{Entscheidung des Leiters der Sonderabteilung der Stalin-Brigade über den Fall des Kompaniechefs Oberleutnant M. Minin vom 7. Februar 1944}

(Herbst 1942 - Februar 1944)

\author{
Bestätige: \\ Stellv. Bevollmächtigter des Zentralstabs \\ der Partisanenbewegung beim Hauptquartier \\ des Oberkommandos \\ für die Oblast' Baranoviči \\ (Dubov)
}

\section{Entscheidung:}

Über die Untersuchung des Falls des Kompaniechefs des Rajonkomitees Ivenec der KP(b)B Oberleutnant Minin, Michail Denisovič, der beschuldigt wird, gesoffen, marodiert, Vertreter der Bevölkerung und Partisanen verprügelt, Frauen vergewaltigt, den Partisanen Gen. Ogurec, Sergej, aus der Ponomarenko-Brigade wie auch die Partisanen einer Kompanie des Rajonkomitees Ivenec der KP(b)B Gen. Migura, Iosif Stanislavovič, und Lipkin, Ivan Fomič, ermordet sowie den Leutnant Gen. Varvarenko aus der Abteilung „Bol'ševik“ der Brigade „Vpered“ tödlich verwundet zu haben.

7. Februar 1944.

Ich, Leiter der Sonderabteilung der Stalin-Brigade Parafenjuk, Vasilij Ivanovič, habe an diesem Tag des laufenden Monats das Untersuchungsmaterial gegen den Kompaniechef des Rajonkomitees Ivenec der KP(b)B, Oblast' Baranoviči der BSSR, Oberleutnant Minin, Michail Denisovič, geprüft, der beschuldigt wird, gesoffen, marodiert, Vertreter der örtlichen Bevölkerung und Partisanen verprügelt, Frauen vergewaltigt, den Partisanen Ogurec, Sergej, aus der Ponomarenko-Brigade wie auch die Partisanen einer Kompanie des Rajonkomitees Ivenec der KP(b)B Gen. Migura, Iosif Stanislavovič, und Lipkin, Ivan Fomič, ermordet sowie den Leutnant Gen. Varvarenko aus der Abteilung „Bol'ševik“ der Brigade „Vpered“ tödlich verwundet zu haben.

Es wurde von mir festgestellt: Oberleutnant der RKKA Minin, Michail Denisovič, geb. 1917, seinen Worten zufolge Mitglied des Komsomol, Nationalität: Belorusse, Hochschulbildung, Zivilberuf: Buchhalter, geboren in Bessarabien, lebte in der Oblast' Mogilev, im Dorf Dory (Rajon Mogilevskij, Taranovskij-Dorfsowjet). Bei den Partisanen ab Juli 1942. Kam in die Oblast' Baranoviči im Auftrag der RKKA-Leitung zur Diversionsarbeit im Hinterland der deutschen Besatzer.

Hier, im gegnerischen Hinterland, hat Minin, M. D., die Sache der Befreiung der Sowjetheimat von den deutschen Besatzern verraten und den Weg des Banditentums und Marodierens eingeschlagen.

Seit der Zeit, da sich im Herbst 1942 kleine Partisanengruppen zu größeren Einheiten - Partisanenabteilungen und -brigaden - vereinigten, gehörte Minin, M. D., der Brigade „Marschall der UdSSR Gen. Žukov“ an. Das Brigadekommando beauftragte Minin, unter der örtlichen Jugend für den Eintritt in die Abteilungen der $\breve{Z} u-$ kov-Brigade zu werben. Nach Rekrutierung von 29 Personen wollte er sich dem Brigadekommando nicht unterordnen, nannte seine Gruppe eine Abteilung und 
ging ans Marodieren und ins Banditentum. Das Brigadekommando verfügte, die Minin-Gruppe zu entwaffnen und Minin als Verräter zu erschießen. Bei der Entwaffnung seiner Gruppe leistete er bewaffneten Widerstand. Als er sah, daß seine Lage ausweglos war, ließ er seine Gruppe im Stich und flüchtete sich mit drei Gesinnungsgenossen, worauf er am 29. April 1943 zur Dzeržinskij-Abteilung der StalinBrigade stieß.

Gemäß einer Übereinkuft zwischen der Leitung der Dzeržinskij-Abteilung und der der Žukov-Brigade mit der Leitung des Vereinigten Rajonzentrums Ivenec wurde Minin am Leben gelassen. Er versprach, die Schande im Kampf gegen die faschistischen Okkupanten zu tilgen. Er wurde in der Dzeržinskij-Abteilung belassen, aber auf dem Rückweg ergab sich Minin dem Suff, erpreßte die Bevölkerung, vergewaltigte Frauen, ließ Bauernpferde in die Stadt Minsk zum Verkauf transportieren, um sich den Erlös aus dem Verkauf der Pferde anzueignen.

Aufgrund der eingegangenen Materialien leitete der Bevollmächtigte der Sonderabteilung der Dzeržinskij-Abteilung Leutnant Gen. Smirnov eine Untersuchung gegen Minin ein. Die Untersuchung erkannte Minin auf schuldig. Gen. Smirnov verlangte am 19. 7. 1943, Minin, M. D., wegen Saufgelagen, Grobheit gegenüber der Bevölkerung und verweigerter Besserung seines Verhaltens vom Posten des Kompaniechefs abzusetzen und den Fall zur weiterer Untersuchung an die Sonderabteilung der Stalin-Brigade weiterzuleiten.

Die Sonderabteilung der Stalin-Brigade setzte die Untersuchung des Falls Minin nicht fort, weil eine deutsche Strafexpedition die Nalibockaja Pušča blockierte. Minin blieb nach wie vor in der Dzeržinskij-Abteilung. Nach der Blockade der Pušča befand sich Minin mit einer Gruppe von Partisanen der Dzeržinskij-Abteilung im Dzeržinskij Rajon, wo er wieder soff, Unfug trieb und zwei Partisanen mit einem Stock verprügelte. Wegen dieser wiederholten Verbrechen wurde Minin von der Leitung der Dzeržinskij-Abteilung vom Posten des Kompaniechefs abgesetzt und aus der Abteilung entlassen (faktisch vertrieben).

Nach der Vertreibung aus der Dzeržinskij-Abteilung erbat sich Minin, M. D., ein Gespräch mit dem Sekretär des vereinigten Rajonzentrums Ivenec, versprach dort, sein Verhalten zu ändern, mit dem Saufen und den Skandalen aufzuhören, und wurde zum Kompaniechef des vereinigten Rajonzentrums Ivenec ernannt. Doch auf diesem Posten gab er das Saufen, die Skandale, die Verprügelung von Partisanen seiner Kompanie und anderer Abteilungen nicht auf.

Am 14. November 1943, als er sich im Ort Rubeževiči des Rajons Ivenec, Oblast' Baranoviči, befand, marodierte er in betrunkenem Zustand. Auf die Forderung der Partisanen der Abteilung „Bol'ševik“ der Brigade „Vpered“, damit aufzuhören, befahl er seiner Gruppe, auf sie zu schießen. Bei der Schießerei wurde Leutnant Gen. Varvarenko aus der Abteilung „Bol’ševik“ der Brigade „Vpered“ tödlich verwundet. Minin wurde auf der Stelle entwaffnet.

Am 30. Dezember 1943 provozierte Minin im Dorf Pil'nica, Rajon Ivenec, eine Schlägerei zwischen den ihm unterstellten Partisanen, den Partisanen der Abteilung "Bol’ševik“ der Stalin-Brigade und den Partisanen der Ponomarenko-Brigade. Die Schlägerei artete in ein richtiggehendes Gefecht aus Gewehren und Maschinenpistolen aus. Ergebnis: Minin verprügelte einen Partisanen aus der Dzeržinskij-Brigade, den Späher der Abteilung „Bol’ševik“ der Stalin-Brigade Gen. Devočko und schoß auf den Aufklärer Levko, Artem. Auch feuerte er auf Hauptmann Šipulin 
und verprügelte den stellvertretenden Bevollmächtigten der Abteilung „Bol'ševik“ der Stalin-Brigade Gen. Leutnant Janušenok. Nach dieser Schlägerei verließ Minin mit seiner Gruppe das Dorf Pil'nica, bezog hinter dem Dorf eine Verteidigungstellung, um den Kampf zu führen, schoß auf das Dorf Pil'nica aus allen in der Gruppe vorhandenen Waffen, auf die Partisanen der Abteilung „Bol’ševik“, der Stalin-Brigade und die Partisanen der Ponomarenko-Brigade. Zuletzt vervollständigte Minin seine verbrecherischen Handlungen an jenem Tag noch dadurch, daß er den Partisanen Gen. Ogurec, Sergej, aus der Ponomarenko-Brigade grausam erwürgte.

Die Untersuchung dieses provokatorischen Falles führte der Leiter der Sonderabteilung der Ponomarenko-Brigade Gen. Sadovskij, D. S., durch. Die Untersuchung stellte fest, daß Minin, M. D., an allen Vorkommnissen des Vorfalls im Dorf Pil'nica am 30. Dezember 1943 schuld war.

Aufgrund der von der Untersuchung festgestellten Fakten stellte der Leiter der Sonderabteilung der Ponomarenko-Brigade, Gen. Sadovskij, D. S., in seinem Gutachten zum Fall des Mordes am Partisanen Ogurec, Sergej, und zu allen Vorkommnissen der provokatorischen Handlungen am 30. Dezember 1943 den Antrag, Minin zur Höchststrafe - zur Erschießung - zu verurteilen.

Aber trotz der so schweren Verbrechen, die Minin während seines Aufenthaltes im gegnerischen Hinterland begangen hatte, durfte er am Leben bleiben; ihm wurde die Möglichkeit gegeben, die furchtbare Schande seiner Verbrechen an der Heimat im Kampf gegen die deutschen Besatzer zu tilgen. Darüber, daß er dieses ihm zur Schande gereichende Mal tilgen werde, gab Minin am 5. Januar 1944 ein schriftliches, von ihm unterschriebenes Versprechen. „Er werde niemals mehr Schnaps trinken und unter den russischen Partisanen im Rajon randalieren, und der Zivilbevölkerung gegenüber werde er sich gut, höflich und kulturell verhalten. Alle Befehle werde er widerspruchslos ausführen."

Zur letzten Bewährung wurde Minin, M. D., auf seinem Posten des Zugführers belassen.

Aber trotz der dreimaligen Entscheidungen der Untersuchungsorgane, daß Minin, M. D., wegen seines Banditentums, der Provokationen gegen die Zivilbevölkerung und die Partisanen zu erschießen sei, trotz seines eigenhändig unterschriebenen Versprechens soff, erpreßte und marodierte er weiter und hielt junge Partisanen dazu an.

Am 29. Januar 1944 provozierte er in betrunkenem Zustand auf dem MikuličiGehöft des Rajons Ivenec einen Schusswechsel mit den Partisanen der Abteilung „Bol'ševik“ der Stalin-Brigade und tötete bei dem Scharmützel mit einer Maschinenpistole eigenhändig den Partisanen der eigenen Kompanic Migura, Iosif Stanislavovič, während Lipkin, Ivan Fomič, im Feuergefecht mit den Partisanen der Abteilung „Bol'ševik“ getötet wurde.

Was den Mord an den Partisanen seiner Kompanie Migura und Lipkin betrifft, so meldete Minin dem Rajonparteikomitee, sie seien von Hauptmann Šipulin aus der Abteilung „Bol'ševik“ der Stalin-Brigade erschossen worden. Somit schlug Minin, M. D., den Weg der Lüge und Provokation ein, weil sich Hauptmann Šipulin nicht am Ort des Vorkommnisses befand. Minin verbot es seinem Partisanen Gen. Doščaško, wahrheitsgetreu vom Verhalten seines Kommandeurs (Minin) zu erzählen.

In Bezug auf den letzten Zwischenfall stellte die Untersuchung fest: Aus dem Dorf Lučicy des Dzeržinskij Rajons kam Minin mit seiner Gruppe von Partisanen 
(Miguro, Lipkin, Doščačko) und in Begleitung einer Frau, genannt Simka, und eines Dorfmusikanten aus Lučicy am 29. Januar 1944 zum Mikuliči-Gehöft, Rajon Ivenec, extra um bei einer Hochzeit zu saufen. Minin und seine Gruppe veranstalteten zweimal ein Saufgelage im Haus der Frau und dreimal im Haus der Braut. Der Partisan dieser Gruppe, Lipkin, I. F., beging mehrere Verbrechen in Form des Marodierens (erpreßte Schnaps im Haus des Bräutigams und im Haus der Braut). Im Haus des Bräutigams nahm er den Hochzeitskuchen mit, und als er unterwegs dem Hochzeitszug begegnete, nahm er das beste Pferd mit der Kutsche weg, wobei er die aus der katholischen Kirche zurückfahrenden Gäste wegjagte und mit der weggenommenen Kutsche spazieren fuhr. Den Hochzeitsmusikanten nahm er die Schellentrommel weg. Dieser Unfug geschah vor den Augen des Kompaniechefs Minin, M. D., der den verbrecherischen Handlungen Lipkins nicht entgegentrat.

Als Minin im Haus des Bräutigams vom Tisch aufstand, schlug er ohne jeden Grund das Tafelgeschirr kaputt.

Am 24. Januar 1944 kam es gegen Abend im Haus des Bräutigams Volochonovič zu einer zweiten Begegnung der Gruppe Minins mit der Gruppe der Partisanen aus der Abteilung „Bol'ševik“ der Stalin-Brigade. Ins Haus von Volochonovič kamen die Partisanen Lipaev, V. K., und Komendantov, A. A., während der Älteste der Gruppe Gen. Stul'ev, E. N., ins Haus des Bürgers Dovnar ging.

Als die Partisanen Lipaev und Komendantov das Haus von Volochonovič betraten, lag Minin im Bett, während Lipkin die Hausbewohner belästigte und von ihnen Schnaps verlangte. Lipaev kam auf Lipkin zu, fragte, worum es ging, und sagte Lipkin, er solle dem Unfug ein Ende machen. Als Antwort auf Lipaevs Forderung schoß Lipkin zweimal aus einer Pistole auf ihn, traf ihn aber nicht, und dann sprang Minin aus dem Bett, eine Maschinenpistole in der Hand, und feuerte auf den Partisanen Komendantov. Diesem gelang es, durch die Tür zu entkommen. Als der Partisan Miguro aus Minins Gruppe die Schüsse hörte, lief er ins Haus hinein, das war während Minins zweiter Salve, und Miguro wurde getötet. Nach dem Mord an Miguro liefen Lipkin und Minin in den Hof hinaus. Minin schoß auf die weglaufenden Partisanen der Abteilung „Bol'ševik“. Bei dem Schusswechsel wurde der Partisan Lipkin aus Minins Gruppe tödlich verwundet und starb an der erlittenen Verletzung. Im Moment der Verwundung hielt Lipkin noch den Hochzeitskuchen, den er Volochonovič gewaltsam weggenommen hatte, in den Händen und hatte zwei Flaschen Schnaps in den Taschen.

Minin und der letzte Partisan seiner Gruppe Doščaško liefen vom Haus von Volochonovič weg, Minin schoß zweimal aus seiner Maschinenpistole auf die Partisanen der Abteilung „Bol'ševik“. Nach den Salven verschwand Minin in unbekannter Richtung und kam nicht einmal zurück, um als Kommandeur zu erfahren, in welchem Zustand seine Kämpfer Miguro und Lipkin waren.

Miguro und Lipkin wurden erst am 30. Januar 1944 unter Beteiligung der Partisanen aus der Abteilung „Bol'ševik“ der Stalin-Brigade begraben.

Bei der Untersuchung in der Sache des Mordes an Miguro und Lipkin bestritt Minin, M. D., seine Schuld, ebenso wie in beiden vorausgehenden Prozessen, und gab nur kleine Verbrechen zu.

Wie die Untersuchung festgestellt hat, zieht sich seit Minins Eintritt in die StalinBrigade (29. April 1943) und durch seinen weiteren Dienst im vereinigten Rajonzentrum Ivenec eine ununterbrochene Kette von antisowjetischen, dem Geist des 
Partisanenkampfes fremden Verbrechen: von Saufgelagen, Skandalen, Schlägereien mit der örtlichen Bevölkerung und mit Partisanen, von Vergewaltigungen, Ausplünderung der Bevölkerung und Transport geraubter Pferde in die Stadt Minsk zum Verkauf und Aneignung des Erlöses, bis zur Provokation bewaffneter Zusammenstöße zwischen den Partisanen: am 14. November 1943 in der Ortschaft Rubeževiči, am 30. Dezember 1943 im Dorf Pil'nica, am 29. Januar 1944 auf dem Gehöft Mikuliči des Rajons Ivenec im Haus von Volochonovič. Infolge der provokatorischen Zusammenstöße, deren Initiator Minin war, wurde der Leutnant Varvarenko tödlich verwundet, der Partisan der Ponomarenko-Brigade Gen. Ogurec, Sergej, grausam erwürgt, der Partisan von Minins eigener Kompanie Miguro getötet und infolge der ebenfalls von Minin provozierten Schießerei der Partisan seiner Kompanie Lipkin getötet. Seine Banditenmethoden brachte er auch den ihm unterstellten Partisanen bei. Davon, daß Minin ein Marodeur war, zeugt das bei ihm aufgefundene katholische Osterkreuz mit einer Perlenkette (liegt der Akte bei).

1. Der Oberleutnant Minin, Michail Denisovič, ist als Marodeur, Bandit und Urheber bewaffneter Zusammenstöße zwischen den Partisanen verschiedener Abteilungen, als unmittelbar am Tod von vier Partisanen Schuldiger zu ERSCHIESSEN.

2. Der Partisan Doščaško, F. B., ist wegen seiner falschen Aussagen beim Verhör vom 1. 2. 1944 für drei Tage in Haft zu nehmen.

Leiter der Sonderabteilung der Stalin-Brigade (Parafenjuk)

F. 1329, op. 1, d. 33, 11. 18-21.

\section{Dokument Nr. 11}

Information des Leiters der Sonderabteilung der Stalin-Brigade vom 9. Februar 1944 über die Untersuchung des Falls des Mordes am Stabschef der Kirov-Abteilung, Hauptmann T. Šemetko

Streng gebeim

An den Oberstleutnant der Staatssicherbeit Gen. Donskoj.

Kopie: An den Sekretär des Rajonkomitees Ivanevskij der KP(b)B Gen. Dubov

Zusammenfassender Bericht

Über den Fall des Mordes am Stabschef

der Kirov-Abteilung der Stalin-Brigade,

Hauptmann Šemetko, Taras Ignat'evič

Die Sonderabteilung der Stalin-Brigade führt eine Untersuchung des Mordes durch, den der stellvertretende Kommandeur der Kirov-Abteilung Major Beljakov am 20. Januar 1944 am Stabschef derselben Abteilung Hauptmann Šemetko, T. I., im Dorf Zaberez', Rajon Juratiški, begangen hat.

Die Untersuchung hat ergeben:

1. Zwischen Major Beljakov und Hauptmann Šemetko bestanden in der Arbeit keine kameradschaftlichen Beziehungen, zweimal kam es zu einem Streit zwischen ihnen. 
2. Am Morgen des 20. Januar betranken sich beide, Beljakov und Šemetko, bei der Bürgerin Baran, N. Ju., im Ort Bakšty. Während der Saufgelage erlaubte sich Hauptmann Šemetko freche Äußerungen über Major Beljakov.

3. Anstatt die Ausreise der ganzen Gruppe ins Lager zu organisieren, ließen Beljakov und Šemetko die ihnen unterstellte Partisanengruppe im Stich und fuhren zu Frauen: Beljakov begab sich ins Dorf Zaberez', Šemetko ins Dorf Paceviči.

4. Auf dem Weg nach Pazeviči machte Šemetko einen Abstecher nach Zaberez', zum Bürger Kalavič, und veranstaltete dort einen Radau, was seinen Ausdruck darin fand, daß Hauptmann Šemetko, der schon betrunken war, beim Bürger Kalavič Schnaps und Patronen verlangte, wobei er ihn mit dem Revolver und dem Bajonett bedrohte. Die Untersuchung hat ergeben, daß Hauptmann Šemetko dem Bürger Kalavič keine Schläge versetzte.

5. Major Beljakov, der mit einer Gruppe von Partisanen eintraf und den Radau im Hause von Kalavič hörte, ging ins Haus und sah dort Šemetko. Beljakov forderte Šemetko auf, ins Lager zu fahren, doch Šemetko weigerte sich.

Laut Zeugenaussagen richtete Hauptmann Šemetko bei diesem Gespräch seinen Revolver gegen Beljakov. Aber Beljakov, der alle Möglichkeiten hatte, Šemetko zu entwaffnen, tötete ihn durch drei Revolverschüsse. Der erste Schuß wurde aus nächster Nähe abgegeben, nach dem zweiten fiel Šemetko hin, und Beljakov gab auf den schon am Boden liegenden Šemetko noch zwei Kopfschüsse ab.

Beljakov verübte den Mord an Šemetko, ohne einer tatsächlichen Lebensgefahr ausgesetzt zu sein. Beljakov hatte eine Gruppe von Partisanen zu seiner Verfügung, er und seine Partisanen befanden sich in unmittelbarer Nähe Šemetkos und hatten alle Möglichkeiten, ihn zu entwaffnen.

Beljakov nahm Šemetkos Gewehr, seine Dokumente und 40 Rubel in Goldmünzen aus der Zarenzeit an sich und ließ die Leiche daliegen.

6. Im Verlauf der Untersuchung habe ich mich davon überzeugt, daß Hauptmann Šemetko vorsätzlich getötet wurde, was ich aus folgenden Fakten schließe:

1) Die Leitung der Kirov-Abteilung nutzte den Umstand aus, daß die Untersuchung am Ort des Mordes durchgeführt wurde, um Beljakov und die Zeugen am 22. Januar, ohne daß es wirklich nötig gewesen wäre, für eine längere Zeit in den Rajon abzukommandieren, wodurch sie die Durchführung der Untersuchung auf frischer Tat faktisch vereitelte.

2) Am 3. Januar 1944 wiesen ich und mein Gehilfe Gen. Ključnikov sowie das Brigadekommando den Kommandeur der Kirov-Abteilung Gen. Griščuk und den Abteilungskommissar Gen. Babenko an, Beljakov und die Personen, die bei dem Mord anwesend waren, sofort zu uns zu beordern; das tat das Abteilungskommando jedoch nicht.

3) Beljakov kehrte am 13. Januar 1944 aus dem Rajon zurück. Als einige Zeugen und Beljakov selbst verhört wurden, entsandte die Leitung der Kirov-Abteilung den Partisanen Ležnevskij, der zur Zeit von Beljakovs Mord an Šemetko dabei gewesen war, in den Rajon, um die Untersuchung zu verschleppen.

7. Die Leitung der Kirov-Abteilung verausgabte die Goldmünzen, die Beljakov Šemetko weggenommen hatte, für persönliche Bedürfnisse: Es wurden ein Stück Tuchstoff für einen Anzug und der Stoff für die Uniform des Abteilungskommandeurs, Papirossy, Gesichtswasser und sonstige Kleinigkeiten gekauft. 
Die Goldmünzen sind nach Beljakovs Aussagen angeblich bei einem Bürger im Dorf Traby beschlagnahmt worden, wo sie eine Durchsuchung durchgeführt und die Münzen in einer Damenhose eingenäht gefunden hätten.

8. Daß der Mord an Šemetko vorsätzlich war, schließe ich aus einer Aussage Beljakovs selbst, da er der Untersuchung erklärte: „Ich habe ihn mit dem zweiten Schuß erledigt, damit er nicht mehr lebte."

Leiter der Sonderabteilung der STALIN-Brigade (Parafenjuk)

9. Februar 1944

F. 1329 , op. 1, d. 33, 11. 31-32.

\title{
Dokument Nr. 12
}

\begin{abstract}
Anordnung des Bevollmächtigten der Sonderabteilung der Stalin-Brigade über die Ausfertigung von Dokumenten für der Erschießung unterliegende Personen aus der Zivilbevölkerung (Februar 1944)
\end{abstract}

Streng gebeim

An die Bevollmächtigten der Abteilungen der STALIN-Brigade

Kopie: An den Oberstleutnant der Staatssicherbeit Gen. Donskoj

Kopie: An den Sekretär des Rajonkomitees Ivenec der KP(b)B Gen. Dubov

In letzter Zeit sind Fälle zu beobachten, wo einzelne Partisanengruppen und einzelne Kommandeure nach eigenem Ermessen Personen aus der Zivilbevölkerung erschießen und die Dokumente über die Erschossenen den Mitarbeitern der Sonderabteilungen zur Dokumentierung der Erschießung zuleiten.

Im Februar 1944 erschoß eine Gruppe von Partisanen aus der Suvorov-Abteilung der Stalin-Brigade den Bürger Kopač, Iosif Nikolaevič, wohnhaft im Dorf Mannukeviči. Vor kurzem erschoß eine Gruppe von Partisanen der Kirov-Abteilung der Stalin-Brigade den 15-jährigen Jungen Audžavicius, worauf die persönlichen $\mathrm{Pa}$ piere des letzteren über den Brigadekommissar zur Ausfertigung der Dokumente über die Erschießung der Sonderabteilung der Brigade zugeleitet wurden.

In solchen Fällen ordne ich an, sich von folgendem leiten zu lassen:

Die Mitarbeiter der Sonderabteilungen fertigen die Dokumente für Personen aus, die unter Teilnahme oder im Auftrag der Mitarbeiter der Sonderabteilungen erschossen werden.

Für Personen, die ohne Teilnahme von Mitarbeitern der Sonderabteilungen erschossen werden, müssen diejenigen die Dokumente ausfertigen, die die ErschieBung vorgenommen haben, und Berichte dem Stab der Abteilung und der Brigade vorlegen, wobei sie die Dokumente in gebührender Weise auszufertigen und darin unter Angabe aller persönlichen und demografischen Auskünfte zu fixieren haben, wer und weswegen erschossen wurde.

Ich mache die Bevollmächtigten der Sonderabteilungen darauf aufmerksam, daß sich nach Rückkehr der Organe der Sowjetmacht jeder von ihnen unter Vorlegen von Dokumenten für jeden Erschossenen wird verantworten müssen. 
Die Mitarbeiter der Sonderabteilungen werden verpflichtet, alle sowohl unmittelbar oder von einzelnen Partisanen als auch durch Gruppen von Partisanen und ihrer Kommandeure Erschossenen zu berücksichtigen.

In den Dokumenten, die bei einer Erschießung ausgefertigt werden, sind alle persönlichen und demografischen Angaben anzuführen.

Leiter der Sonderabteilung der STALIN-Brigade Februar 1944

(Parafenjuk)

F. 1329, op. 1, d. 33, 1. 30 .

\section{Dokument Nr. 13}

\section{Prüfungsbericht des Leiters der Operativen Tschekistengruppe der Lida-Zone vom 2. Juni 1944 über den Fall der Erschießung des Hauptfeldwebels V. Korenevskij aus der Kotovskij-Abteilung der Dzeržinskij-Brigade}

Gehilfe des Bevollmächtigten des Stabs

der Partisanenbewegung und des $\mathrm{ZK}$ der $\mathrm{KP}(\mathrm{b}) \mathrm{B}$

für die Oblast' Baranoviči

(Sokolov)

\section{Prüfungsbericht:}

Über den Fall der Erschießung des ehem. Hauptfeldwebel

der Kotovskij-Abteilung der Dzeržinskij-Brigade

Korenevskij, Vasilij Vasil'evič

2. Juni 1944

Ich, Major der Staatssicherheit VALERIJ, Leiter der Operativen Gruppe der Lida-Zone, habe die Untersuchungsmaterialien der Dzeržinskij- und der Čapaev-Partisanenbrigade zum Fall der Erschießung des Hauptfeldwebel Korenevskij, V. V., geprüft und

\section{BEFUNDEN:}

Um 2 Uhr am 19. Mai 1944 kam der Hauptfeldwebel der Kotovskij-Abteilung Korenevskij, V. V., in betrunkenem Zustand ins Dorf Ljachoviči-Vtorye und ging ins Haus von Ermolovič, wobei er unterwegs den Anruf des Postens nicht beachtete und zwei Schüsse abgab.

In der Wohnung von Ermolovič benahm sich Korenevskij taktlos (war grob, erlaubte sich Handgreiflichkeiten u.a.); das stellten folgende Personen fest, die am Ort der Begebenheit eingetroffen waren: der Leiter der Sonderabteilung der Čapaev-Partisanenabteilung Bus'ko, der stellvertretende Brigadekommissar Chomičev, der Bevollmächtigte der Sonderabteilung der Furmanov-Abteilung Kireev, T., der Kommandant des Dorfes Ljachoviči Zubrik sowie die Bewohner Ermolovič, Ivan, und Ermolovičeva, Agaf'ja.

Anstatt Korenevskij zu entwaffnen und in die Kotovskij-Partisanenabteilung zur Bestrafung zu transportieren, ließ sich der Leiter der Sonderabteilung der Partisanenbrigade Bus'ko in lange Streitereien und Fragereien mit Korenevskij ein, und schließlich tötete der Bevollmächtigte der Sonderabteilung der Furmanov-Partisa- 
nenabteilung Kireev, T., Korenevskij durch einen Pistolenschuß, wodurch er ein erhebliches Vergehen beging.

Ich betrachte diesen Fall als einen ungerechtfertigten Schuß, man hätte (die Partisanenbrigade war durch vier Personen vertreten) Korenevskij entwaffnen und in Haft nehmen können, aber das taten der Leiter der Sonderabteilung Bus'ko und der Bevollmächtigte der Sonderabteilung der Furmanov-Partisanenabteilung Kireev nicht.

Aufgrund der Materialien stelle ich fest, daß Korenevskij grobe Disziplinarvergehen zugelassen, auf den Anruf des Postens nicht reagiert und sich dem Leiter der Sonderabteilung der Brigade nicht untergeordnet hatte, weswegen er eine Strafe hätte ertragen sollen, aber nicht das Strafmaß, für welches sich der Bevollmächtigte Kireev entschloß.

Anhand des Materials ist festgestellt worden, daß der Kommandeur der ČapaevBrigade Kudrin diesen Fall vertuscht und einseitig nur Korenevskij beschuldigt, keineswegs dagegen Bus'ko und Kireev, welch letzterer Korenevskij erschossen hat.

Ausgehend vom oben Dargelegten, befinde ich, daß

1. dem Leiter der Sonderabteilung der Čapaev-Partisanenbrigade Gen. Bus'ko eine strenge Rüge wegen nicht operativer Handlungsweise und einer ebensolchen Erfüllung seiner Pflichten zu erteilen ist;

2. der Bevollmächtigte der Sonderabteilung der Furmanov-Partisanenbrigade Kireew, T., für 10 Tage zu verhaften, vom Posten des Bevollmächtigten abzusetzen und zu den Mannschaften zu überführen ist;

3. dem Kommandeur der Čapaev-Brigade Gen. Kudrin ein Verweis zu erteilen und daß er zu verwarnen ist, damit er in Zukunft solche Vorkommnisse nicht zuläßt und jeden Fall allseitig prüft.

Leiter der Operativen Tschekistengruppe

der Lida-Zone

Major der Staatssicherheit 